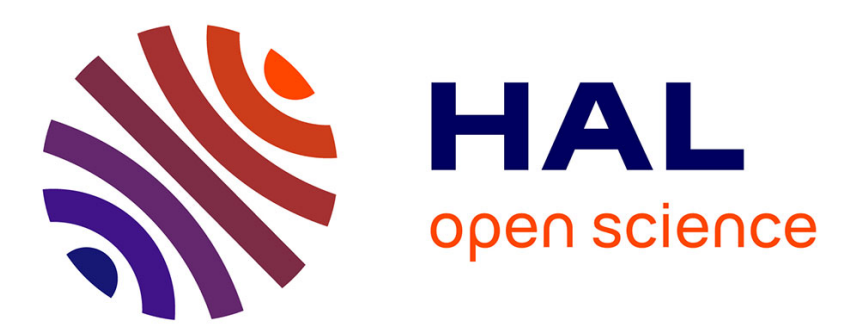

\title{
Post-buckling nonlinear static and dynamical analyses of uncertain cylindrical shells and experimental validation
}

Evangéline Capiez-Lernout, Christian Soize, Marc Mignolet

\section{To cite this version:}

Evangéline Capiez-Lernout, Christian Soize, Marc Mignolet. Post-buckling nonlinear static and dynamical analyses of uncertain cylindrical shells and experimental validation. Computer Methods in Applied Mechanics and Engineering, 2014, 271 (1), pp.210-230. 10.1016/j.cma.2013.12.011. hal00922708

\section{HAL Id: hal-00922708 \\ https://hal.science/hal-00922708}

Submitted on 29 Dec 2013

HAL is a multi-disciplinary open access archive for the deposit and dissemination of scientific research documents, whether they are published or not. The documents may come from teaching and research institutions in France or abroad, or from public or private research centers.
L'archive ouverte pluridisciplinaire HAL, est destinée au dépôt et à la diffusion de documents scientifiques de niveau recherche, publiés ou non, émanant des établissements d'enseignement et de recherche français ou étrangers, des laboratoires publics ou privés. 


\title{
Post-buckling nonlinear static and dynamical analyses of uncertain cylindrical shells and experimental validation
}

\author{
E. Capiez-Lernout ${ }^{\star}, \mathrm{a}$, C. Soize ${ }^{\mathrm{a}}$, M.-P. Mignolet ${ }^{\mathrm{b}}$ \\ ${ }^{a}$ Université Paris-Est, Laboratoire Modélisation et Simulation Multi Echelle, MSME \\ UMR 8208 CNRS, 5 bd Descartes, 77454 Marne-la-Vallée Cedex 02, France \\ ${ }^{b}$ Arizona State University, SEMTE, Faculties of Mechanical and Aerospace Engineering, \\ Tempe, Arizona 8528\%-6106, USA
}

\begin{abstract}
The paper presents a complete experimental validation of an advanced computational methodology adapted to the nonlinear post-buckling analysis of geometrically nonlinear structures in presence of uncertainty. A mean nonlinear reduced-order computational model is first obtained using an adapted projection basis. The stochastic nonlinear computational model is then constructed as a function of a scalar dispersion parameter, which has to be identified with respect to the nonlinear static experimental response of a very thin cylindrical shell submitted to a static shear load. The identified stochastic computational model is finally used for predicting the nonlinear dynamical post-buckling behavior of the structure submitted to a stochastic ground motion.
\end{abstract}

Keywords:

uncertainties, non-stationary stochastic excitation, geometrical nonlinearities, static post-buckling, dynamic post-buckling, experimental identification

\section{Introduction}

The focus of this paper is on the post-buckling mechanical behavior of thin cylindrical shells, which is currently a subject of interest for which numerical predictions do not always match experimental measurements. This class of structures is commonly present in industrial equipments, such as silos, tanks for gas and liquid, reactor vessels, etc. The discrepancy between experimental observations and predictions is often due to the particular sensitivity of 
thin cylindrical shell structure to the presence of initial imperfections (heterogeneity of the materials, imperfect boundary conditions, inhomogeneous thickness induced by the manufacturing process and geometry). Note that for cylindrical shells of very small thickness, the geometrically nonlinear effects induced by large strains and large displacements must be taken into account. Numerous sensitivity analyses to standard geometric imperfections can be found in the literature, distinguishing several classes of external loads such as axial compression $[1,2,3]$, pressure load $[4,5]$ and shear load $[6,7,8,9,10]$.

However, a generic sensitivity analysis of such structures with respect to any kind of imperfections requires the introduction of adapted non-deterministic approaches to represent uncertainties. For example, non-probabilistic approaches involving either interval analysis [11] or anti-optimization strategies [12] have been developed in the context of post-buckling analysis of structures. An experimental validation related to the identification of the buckling load of composite cylindrical shells can be found in [13].

Probabilistic approaches have also been used for representing the random uncertainties in the numerical computational models. The problems involving large nonlinear computational models, taking into account either or both the presence of random uncertainties and the stochastic nature of the loading requires appropriate strategies to properly achieve the dynamical analysis, see for instance $[14,15]$. More particularly, nonlinear stochastic buckling analyses have recently been conducted in which geometrical imperfections $[16,17]$ and random boundary conditions [18] were modeled as Gaussian random fields whose statistical properties are issued from available experimental data. Non-Gaussian random fields have also been used for studying the sensitivity of buckling loads with respect to material and geometric imperfections of cylindrical shells [19]. Such probabilistic models of uncertainties will be referred to as parametric here as they focus the uncertainty only on specific aspects/parameters of the computational models selected by the analyst. It is also well known that the geometric imperfections particularly responsible for the sensitiveness of such structures [16, 20].

In the investigation of unstiffened composite cylinders carried out in [17], the knowledge of existing missing composite fibers justified the modeling of material imperfections. Due to the lack of available experimental measurements, material imperfections were taken into account with chosen random properties. Despite the accurate modeling of the geometrical imperfections, the buckling load calculated with the nonlinear stochastic computational model was overestimated suggesting that other uncertainties, not considered 
in the analysis, were present and affected the experimental measurements.

An alternative approach, referred to as the nonparametric probabilistic approach, has been developed for situations in which the uncertainty cannot be singled out in one or a few parameters in the computational model. It allows the consideration of both system-parameter uncertainties and model uncertainties [21] by proceeding at the level of modal/reduced-order models developed on deterministic bases. Note that the nonparametric approach has been extended to uncertain nonlinear reduced-order models of geometrically nonlinear structures [22].

The development of such nonlinear reduced-order models requires first the selection of an appropriate deterministic basis for the representation of the response. This basis can be obtained by one of several techniques such as the Proper Orthogonal Decomposition method (POD method) [23, 24, 25], which is known to be particularly efficient for nonlinear static cases. One can also rely on linear elastic modes of vibration $[26,27]$, or selected linear elastic modes appropriately enriched, e.g. [28], see [29] for a recent review. The parameters of the nonlinear reduced-order model of the mean structure can then be either deduced using the STEP procedure (which is based on the smart non-intrusive use of standard commercial finite element codes) $[30,22,29]$ or from explicit construction as shown in [31] in the context of three-dimensional solid finite elements.

Having established the reduced-order model of the mean structure, uncertainties on the linear and on the nonlinear parts of the stiffness operator are introduced in the nonparametric framework. This is accomplished through the construction of a dedicated random operator with values in the set of all positive-definite symmetric real matrices whose mean value involves all linear, quadratic and cubic stiffness terms of the mean nonlinear reducedorder model [22]. The resulting stochastic nonlinear computational model is characterized by a single scalar dispersion parameter, quantifying the level of uncertainty in the stiffness properties which can easily be identified with experiments. Experimental validations based on this theory can be found in $[31,32]$ for slender elastic bodies, e.g. beams.

The paper is organized as follows. Section 2 summarizes the main steps leading to the mean non-linear reduced-order computational model following the approach of [31]. Section 3 is devoted to the construction of the stochastic nonlinear computational model using the nonparametric probabilistic approach for modeling the random uncertainties. A Gaussian nonstationary second-order stochastic process is also introduced to represent the 
prescribed, earthquake-induced ground motions. An identification effort is carried out in section 4 to calibrate the stiffness dispersion parameter of the stochastic nonlinear computational model from experimental measurements of the response of the cylindrical shell. Finally, the nonlinear post-buckling dynamical analysis of the uncertain cylindrical shell is carried out in Section 5 using the previously identified stochastic nonlinear computational model subjected to the prescribed ground motions.

\section{Reduced-order computational model using 3D elasticity in large deformation}

This Section is devoted to the construction of a nonlinear reduced-order model in the context of elastodynamics involving geometrical nonlinearity.

\subsection{Mathematical notations}

From here on, the convention for summation over repeated indices will be adopted. Let $a(\mathbf{x}, t)$ be a given function. The following notations are used : $a_{, j}=\partial a / \partial x_{j}, \dot{a}=\partial a / \partial t, \ddot{a}=\partial^{2} a / \partial t^{2}$.

\subsection{Description of the nonlinear boundary value problem}

The structure under consideration is composed of a linear elastic material and is assumed to undergo large deformations inducing geometrical nonlinearities. A total Lagrangian formulation is chosen. Consequently, the dynamical equations are written with respect to the reference configuration. Let $\Omega$ be the three-dimensional bounded domain of the physical space $\mathbb{R}^{3}$ corresponding to the reference configuration taken as a natural state without prestress and subjected to the body force field $\mathbf{g}(\mathbf{x}, t)$, in which $\mathbf{x}$ denotes the position of a given point belonging to domain $\Omega$. The boundary $\partial \Omega$ is such that $\partial \Omega=\Gamma \cup \Sigma$ with $\Gamma \cap \Sigma=\emptyset$ and the external unit normal to boundary $\partial \Omega$ is denoted by $\mathbf{n}$. The boundary part $\Gamma$ corresponds to the fixed part of the structure whereas the boundary part $\Sigma$ is subjected to the external surface force field $\mathbf{G}(\mathbf{x}, t)$. The displacement field expressed with respect to the reference configuration is denoted as $\mathbf{u}(\mathbf{x}, t)$. Note that force fields $\mathbf{G}(\mathbf{x}, t)$ and $\mathbf{g}(\mathbf{x}, t)$ correspond to the Lagrangian transport into the reference configuration of the physical surface force field and to the physical body force field applied on the deformed configuration.

The boundary value problem is thus described by the following set of nonlinear equations : 


$$
\begin{aligned}
\left(F_{i j} S_{j k}\right)_{, k}+g_{i} & =\rho \ddot{u}_{i} \quad \text { in } \Omega \\
F_{i j} S_{j k} n_{k} & =G_{i} \quad \text { on } \Sigma \\
u_{i} & =0 \quad \text { on } \Gamma,
\end{aligned}
$$

with the initial conditions given by

$$
u_{i}(\mathbf{x}, 0)=0 \quad, \quad \dot{u}_{i}(\mathbf{x}, 0)=0 \quad .
$$

In Eq.(1), the mass density field $\rho(\mathbf{x})$ is expressed in the reference configuration. The deformation gradient tensor is $\mathbb{E}=\left\{F_{i j}\right\}_{i j}$, whose components $F_{i j}$ are defined by

$$
F_{i j}=u_{i, j}+\delta_{i j}
$$

in which $\delta_{i j}$ is the Kronecker symbol such that $\delta_{i j}=1$ if $i=j$ and $\delta_{i j}=0$ otherwise. The second Piola-Kirchoff symmetric stress tensor $\mathbb{S}=\left\{S_{i j}\right\}_{i j}$ is written, for a linear elastic material, as

$$
S_{i j}=a_{i j k \ell} E_{k \ell}
$$

In Eq.(6), the fourth-order elasticity tensor $\mathbb{a}=\left\{a_{i j k \ell}\right\}_{i j k \ell}$ satisfies the usual symmetry and positive-definiteness properties. The Green strain tensor $\mathbb{E}=$ $\left\{E_{i j}\right\}_{i j}$ is then written as the sum of linear and nonlinear terms such that

$$
E_{i j}=\varepsilon_{i j}+\eta_{i j}
$$

in which

$$
\varepsilon_{i j}=\frac{1}{2}\left(u_{i, j}+u_{j, i}\right) \quad \text { and } \quad \eta_{i j}=\frac{1}{2} u_{s, i} u_{s, j}
$$

2.3. Weak formulation of the nonlinear boundary value problem

2.3.1. Case of a Dirichlet condition on boundary $\Gamma$

Let $\mathcal{C}$ be the admissible space defined by

$$
\mathcal{C}=\{\mathbf{v} \in \Omega, \mathbf{v} \text { sufficiently regular }, \mathbf{v}=\mathbf{0} \text { on } \Gamma\} .
$$

Considering time $t$ as a parameter, the weak formulation of the nonlinear boundary value problem described by Eqs. (1) to (3) consists in finding the 
unknown displacement field $\mathbf{u}(\cdot, t) \in \mathcal{C}$ such that, for any admissible displacement field $\mathbf{v}$ in $\mathcal{C}$

$$
m(\ddot{\mathbf{u}}, \mathbf{v})+d(\dot{\mathbf{u}}, \mathbf{v})+k^{(1)}(\mathbf{u}, \mathbf{v})+k^{(2)}(\mathbf{u}, \mathbf{u}, \mathbf{v})+k^{(3)}(\mathbf{u}, \mathbf{u}, \mathbf{u}, \mathbf{v})=f(\mathbf{v}),
$$

with the initial conditions given by Eq.(4) and where,

$$
\begin{aligned}
& f(\mathbf{v})=\int_{\Omega} v_{i} g_{i} d \mathbf{x}+\int_{\Sigma} v_{i} G_{i} d \mathbf{s} \\
& m(\ddot{\mathbf{u}}, \mathbf{v})=\int_{\Omega} \rho \ddot{u}_{i} v_{i} d \mathbf{x} \\
& k^{(1)}(\mathbf{u}, \mathbf{v})=\int_{\Omega} a_{j k \ell m} \varepsilon_{\ell m}(\mathbf{u}) \varepsilon_{j k}(\mathbf{v}) d \mathbf{x} \\
& k^{(2)}(\mathbf{u}, \mathbf{u}, \mathbf{v})=\int_{\Omega} a_{j k \ell m} \eta_{\ell m}(\mathbf{u}) \varepsilon_{j k}(\mathbf{v}) d \mathbf{x}+\int_{\Omega} a_{j k \ell m} u_{s, j} v_{s, k} \varepsilon_{\ell m}(\mathbf{u}) d \mathbf{x}(1) \\
& k^{(3)}(\mathbf{u}, \mathbf{u}, \mathbf{u}, \mathbf{v})=\int_{\Omega} a_{j k \ell m} u_{s, j} v_{s, k} \eta_{\ell m}(\mathbf{u}) d \mathbf{x}
\end{aligned}
$$

In Eq.(10), a usual linearized viscous term represented by the positive-definite symmetric bilinear form $d(\mathbf{u}, \mathbf{v})$ is added in order to model the dissipation effects occurring in the structure [33].

\subsubsection{Case of a ground motion on boundary $\Gamma$}

From here on, the earthquake engineering context is investigated. The boundary $\Gamma$, which was assumed at rest above, experiences a rigid body displacement induced by a prescribed based motion $\mathbf{u}_{\Gamma}(\mathbf{x}, t)=\mathbf{u}_{\Gamma}(t), \forall \mathbf{x} \in \Gamma$. The corresponding displacement field of the structure can then be expressed as $\mathbf{u}_{\mathrm{exc}}(\mathbf{x}, t)+\mathbf{u}(\mathbf{x}, t)$ where

$m(\ddot{\mathbf{u}}, \mathbf{v})+d(\dot{\mathbf{u}}, \mathbf{v})+k^{(1)}(\mathbf{u}, \mathbf{v})+k^{(2)}(\mathbf{u}, \mathbf{u}, \mathbf{v})+k^{(3)}(\mathbf{u}, \mathbf{u}, \mathbf{u}, \mathbf{v})=f(\mathbf{v})-m\left(\ddot{\mathbf{u}}_{\mathrm{exc}}, \mathbf{v}\right)$

in which $\mathbf{u}_{\text {exc }}(\mathbf{x}, t)=\mathbf{u}_{\Gamma}(t), \forall \mathbf{x} \in \Omega$.

\subsection{Mean nonlinear reduced-order model}

Let $\varphi^{\alpha}(\mathbf{x}), \alpha=\{1, \ldots, N\}$, be a finite family of an orthonormal vector basis of $\mathcal{C}$ verifying the following property

$$
\int_{\Omega} \varphi_{i}^{\alpha} \varphi_{i}^{\beta} d \mathbf{x}=\delta_{\alpha \beta}
$$


The vector $\mathbf{q}=\left(q_{1}, \ldots, q_{N}\right)$ of the corresponding generalized coordinates is introduced as a new set of unknown variables by projecting the reference nonlinear response $\mathbf{u}(\mathbf{x}, \cdot)$ on the vector space spanned by $\left\{\varphi^{1}, \cdots, \varphi^{N}\right\}$. The approximation $\mathbf{u}^{N}(\mathbf{x}, t)$ of order $N$ of $\mathbf{u}(\mathbf{x}, t)$ is then written as

$$
\mathbf{u}^{N}(\mathbf{x}, t)=\sum_{\beta=1}^{N} \varphi^{\beta}(\mathbf{x}) q_{\beta}(t)
$$

Taking the test function $\mathbf{v}$ as $\varphi^{\alpha}$ in Eq.(16) and using Eq.(18) yields

$$
\mathcal{M}_{\alpha \beta} \ddot{q}_{\beta}+\mathcal{D}_{\alpha \beta} \dot{q}_{\beta}+\mathcal{K}_{\alpha \beta}^{(1)} q_{\beta}+\mathcal{K}_{\alpha \beta \gamma}^{(2)} q_{\beta} q_{\gamma}+\mathcal{K}_{\alpha \beta \gamma \delta}^{(3)} q_{\beta} q_{\gamma} q_{\delta}=\mathcal{F}_{\alpha},
$$

in which

$$
\begin{gathered}
\mathcal{K}_{\alpha \beta}^{(1)}=\int_{\Omega} a_{j k \ell m} \varphi_{j, k}^{\alpha} \varphi_{\ell, m}^{\beta} d \mathbf{x} \\
\mathcal{K}_{\alpha \beta \gamma}^{(2)}=\frac{1}{2}\left(\widehat{\mathcal{K}}_{\alpha \beta \gamma}^{(2)}+\widehat{\mathcal{K}}_{\beta \gamma \alpha}^{(2)}+\widehat{\mathcal{K}}_{\gamma \alpha \beta}^{(2)}\right) \\
\widehat{\mathcal{K}}_{\alpha \beta \gamma}^{(2)}=\int_{\Omega} a_{j k \ell m} \varphi_{j, k}^{\alpha} \varphi_{s, \ell}^{\beta} \varphi_{s, m}^{\gamma} d \mathbf{x}, \\
\mathcal{K}_{\alpha \beta \gamma \delta}^{(3)}=\frac{1}{2} \int_{\Omega} a_{j k \ell m} \varphi_{r, j}^{\alpha} \varphi_{r, k}^{\beta} \varphi_{s, \ell}^{\gamma} \varphi_{s, m}^{\delta} d \mathbf{x} \\
\mathcal{F}_{\alpha}=\int_{\Omega} g_{i} \varphi_{i}^{\alpha} d \mathbf{x}+\int_{\Sigma} G_{i} \varphi_{i}^{\alpha} d \mathbf{s}-\int_{\Omega} \rho\left(\ddot{\mathbf{u}}_{\Gamma}\right)_{i} \varphi_{i}^{\alpha} d \mathbf{x} \\
\mathcal{M}_{\alpha \beta}=\int_{\Omega} \rho \varphi_{i}^{\alpha} \varphi_{i}^{\beta} d \mathbf{x} .
\end{gathered}
$$

The model of the reduced damping operator $\mathcal{D}_{\alpha \beta}$ is chosen here as

$$
\mathcal{D}_{\alpha \beta}=\zeta \mathcal{K}_{\alpha \beta}^{(1)},
$$

in which $\zeta$ is a positive constant. It should be noted that the damping modeling can be changed without difficulties.

The mean (or nominal) nonlinear reduced-order computational model is explicitly defined once the projection basis is chosen. The construction is carried out in the context of the three-dimensional finite element method. The finite elements are isoparametric solid finite elements with 8 nodes and the numerical integration is carried out with 8 Gauss integration points. The main steps of the procedure, which uses the symmetry properties of the reduced operators, can be found in [31] and are summarized below: 
- computation of the elementary contributions of each type of internal forces projected on the vector basis

- finite element assembly of these elementary contributions

- computation of the operators $\mathcal{M}_{\alpha \beta}, \mathcal{D}_{\alpha \beta}, \mathcal{K}_{\alpha \beta}^{(1)}, \widehat{\mathcal{K}}_{\alpha \beta \gamma}^{(2)}$ and $\mathcal{K}_{\alpha \beta \gamma \delta}^{(3)}$ of the mean nonlinear reduced-order model

\section{Uncertainty quantification and stochastic excitation}

In this Section, the nonparametric probabilistic approach of uncertainties is used for modeling the uncertainties in the nonlinear computational model and the earthquake excitation is modeled by a nonstationary stochastic process.

\subsection{Nonparametric stochastic modeling of uncertainties}

\subsubsection{Definition of a reshaped stiffness matrix}

The main idea of the nonparametric probabilistic approach is to replace each of the matrices of a given mean reduced computational model by a random matrix whose probability model is constructed from the maximum entropy principle using the available information [34, 21]. In the present geometrically nonlinear context [22], the nonlinear equations involve nonlinear operators. Let $P=N(N+1)$. The $(P \times P)$ real matrix $[\mathcal{K}]$ is introduced as

$$
[\mathcal{K}]=\left[\begin{array}{cc}
{\left[\mathcal{K}^{(1)}\right]} & {\left[\widehat{\mathcal{K}}^{(2)}\right]} \\
{\left[\widehat{\mathcal{K}}^{(2)}\right]^{T}} & 2\left[\mathcal{K}^{(3)}\right]
\end{array}\right],
$$

in which the matrix blocks $\left[\widehat{\mathcal{K}}^{(2)}\right]$ and $\left[\mathcal{K}^{(3)}\right]$ are the $\left(N \times N^{2}\right)$ and the $\left(N^{2} \times N^{2}\right)$ real matrices resulting from the following reshaping operation

$$
\begin{gathered}
{\left[\widehat{\mathcal{K}}^{(2)}\right]_{\alpha J}=\widehat{\mathcal{K}}_{\alpha \beta \gamma}^{(2)}, \quad J=(\beta-1) N+\gamma,} \\
{\left[\mathcal{K}^{(3)}\right]_{I J}=\mathcal{K}_{\alpha \beta \gamma \delta}^{(3)}, I=(\alpha-1) N+\beta \text { and } J=(\gamma-1) N+\delta .}
\end{gathered}
$$

It has been shown in $[22]$ that the matrix $[\mathcal{K}]$ is a symmetric and positivedefinite matrix. Consequently, the nonparametric probabilistic approach initially introduced in the linear context for positive-definite symmetric operators can easily be extended to the geometrically nonlinear context. 


\subsubsection{Construction of the random matrix model}

The mean reduced matrices $[\mathcal{M}],[\mathcal{D}],[\mathcal{K}]$ are then replaced by the random matrices $[\mathcal{M}],[\mathcal{D}],[\mathcal{K}]$ defined on the probability space $(\Theta, \mathcal{T}, \mathcal{P})$ such that $\mathcal{E}\{[\mathcal{M}]\}=[\mathcal{M}], \mathcal{E}\{[\mathcal{D}]\}=[\mathcal{D}], \mathcal{E}\{[\mathcal{K}]\}=[\mathcal{K}]$ in which $\mathcal{E}$ is the mathematical expectation. The random matrices $[\mathcal{M}],[\mathcal{D}],[\mathcal{K}]$ are then written as $[\mathcal{M}]=\left[L_{M}\right]^{T}\left[\mathbf{G}_{M}\left(\delta_{M}\right)\right]\left[L_{M}\right],[\mathcal{D}]=\left[L_{D}\right]^{T}\left[\mathbf{G}_{D}\left(\delta_{D}\right)\right]\left[L_{D}\right]$, $[\mathcal{K}]=\left[L_{K}\right]^{T}\left[\mathbf{G}_{K}\left(\delta_{K}\right)\right]\left[L_{K}\right]$ in which $\left[L_{M}\right],\left[L_{D}\right]$ and $\left[L_{K}\right]$ are $(N \times N)$, $(N \times N)$ and $(P \times P)$ real upper matrices such that $[\mathcal{M}]=\left[L_{M}\right]^{T}\left[L_{M}\right]$, $[\mathcal{D}]=\left[L_{D}\right]^{T}\left[L_{D}\right]$ and $[\mathcal{K}]=\left[L_{K}\right]^{T}\left[L_{K}\right]$. Further, $\left[\mathbf{G}_{M}\right],\left[\mathbf{G}_{D}\right]$ and $\left[\mathbf{G}_{K}\right]$ are full random matrices with values in the set of the positive-definite symmetric $(N \times N),(N \times N)$ and $(P \times P)$ matrices. The probability distributions and the random generators of random matrices $\left[\mathbf{G}_{M}\left(\delta_{M}\right)\right],\left[\mathbf{G}_{D}\left(\delta_{D}\right)\right]$ and $\left[\mathbf{G}_{K}\left(\delta_{K}\right)\right]$ are constructed in [21]. The level of uncertainty is quantified by the dispersion parameter $\boldsymbol{\delta}=\left(\delta_{M}, \delta_{D}, \delta_{K}\right)$ defined on a subset $\Delta$ of $\mathbb{R}^{3}$.

\subsection{Stochastic excitation}

The generalized external load $\mathcal{F}(t)=\left(\mathcal{F}_{1}(t), \ldots, \mathcal{F}_{N}(t)\right)$ is split into a static load $\mathcal{F}^{\text {stat }}(t)$ and a dynamical one $\mathcal{F}^{\text {dyna }}(t)$ such that

$$
\mathcal{F}(t)=\mathcal{F}^{\text {stat }}(t)+\mathcal{F}^{\text {dyna }}(t)
$$

The dynamical load can be rewritten as

$$
\mathcal{F}_{\alpha}^{\text {dyna }}(t)=-\int_{\Omega} \rho(\mathbf{x})\left(\ddot{\mathbf{u}}_{\mathrm{exc}}(\mathbf{x}, t)\right)_{i} \varphi_{i}^{\alpha}(\mathbf{x}) d \mathbf{x}
$$

and the static load can be deduced from Eq.(24). From here on, it is assumed that the ground motion is along a given direction. Consequently, the acceleration field in Eq.(31) can be written as

$$
\ddot{\mathbf{u}}_{\mathrm{exc}}(\mathbf{x}, t)=\mathbf{w}_{0} \gamma(t)
$$

in which $\mathbf{w}_{0}$ is the unit vector of $\mathbb{R}^{3}$ characterizing the direction of the ground motion and where $\{\gamma(t), t \geq 0\}$ is a real-valued scalar function. Replacing Eq.(32) in Eq.(31) yields $\mathcal{F}^{\text {dyna }}(t)=-\left[L_{M}\right]^{T}[T] \gamma(t)$, in which $[T]$ is the $\mathbb{R}^{N}$-vector solution of

$$
\left\{\left[L_{M}\right]^{T}[T]\right\}_{\alpha}=\int_{\Omega} \rho\left(\mathbf{w}_{0}\right)_{i} \varphi_{i}^{\alpha} d \mathbf{x}
$$


Note that matrix $\left[L_{M}\right]$ is known and invertible. Consequently, Eq.(33) uniquely defines the vector $[T]$. In the earthquake engineering context, the acceleration $\gamma(t)$ induced by the ground motion is replaced by the random quantity $\boldsymbol{\Gamma}(t)$ with values in $\mathbb{R}$. Note that $\mathcal{F}^{\text {dyna }}(t)$ depends on the structural mass distribution. Given the fact that there exists uncertainty on this distribution, the vector $\left[L_{M}\right]^{T}[T]$ should not be considered as a deterministic quantity. Consistently with the invertibility property of matrix $\left[L_{M}\right]$, the dynamical load $\mathcal{F}^{\text {dyna }}(t)$ is modeled by the $\mathbb{R}^{N}$-valued random vector

$$
\mathcal{F}^{\text {dyna }}(t)=-\left[L_{M}\right]^{T}\left[\mathbf{G}_{M}\left(\delta_{M}\right)\right][T] \boldsymbol{\Gamma}(t) .
$$

The acceleration $\{\boldsymbol{\Gamma}(t), t \geq 0\}$ is modeled here by a Gaussian, non-stationary, centered, second-order stochastic process defined on a probability space $\left(\Theta^{\prime}, \mathcal{T}^{\prime}, \mathcal{P}^{\prime}\right)$ which is different from probability space $(\Theta, \mathcal{T}, \mathcal{P})$. Consequently, the stochastic process $\Gamma$ is completely defined by its autocorrelation function $R_{\Gamma}\left(t, t^{\prime}\right)=$ $\mathcal{E}\left\{\boldsymbol{\Gamma}(t) \boldsymbol{\Gamma}\left(t^{\prime}\right)\right\}$. The following usual representation [35] of $\boldsymbol{\Gamma}$ for earthquake accelerograms is adopted

$$
\boldsymbol{\Gamma}(t)=g(t) \boldsymbol{\beta}(t),
$$

in which the function $g(t)$ is the envelope function whose representation can be found in $[35,36,37]$. Further, $\{\boldsymbol{\beta}(t), t \in \mathbb{R}\}$ is a real-valued Gaussian, stationary, centered, second-order stochastic process for which the power spectral density function $S_{\boldsymbol{\beta}}(\omega)$ can be written as a rational function [38]. We then have

$$
R_{\Gamma}\left(t, t^{\prime}\right)=g(t) g\left(t^{\prime}\right) R_{\boldsymbol{\beta}}\left(t-t^{\prime}\right),
$$

where $R_{\boldsymbol{\beta}}\left(t-t^{\prime}\right)$ is the autocorrelation function of stochastic process $\boldsymbol{\beta}(t)$ such that

$$
R_{\boldsymbol{\beta}}\left(t-t^{\prime}\right)=\int_{\mathbb{R}} S_{\boldsymbol{\beta}}(\omega) e^{i \omega\left(t-t^{\prime}\right)} d \omega .
$$

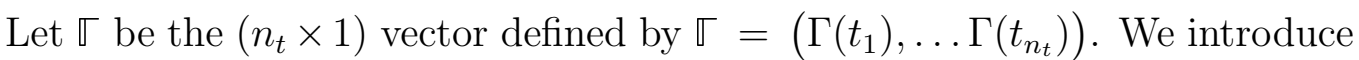
the time sampling $t_{i}=(i-1) \Delta t, i=\left\{1, \ldots, n_{t}\right\}$, of $[0, T]$ with $T=n_{t} \Delta t$. Then denote by $\left[R_{\mathbb{}}\right]=\mathcal{E}\left\{\mathbb{\square} \mathbb{\square}^{T}\right\}$ the correlation matrix of $\mathbb{T}$, which can be evaluated from $R_{\boldsymbol{\beta}}(t)$ of Eq.(37), by Fast Fourier Transform (FFT). The random vector $\llbracket$ can accordingly be written as

$$
\mathbb{}=[L] \mathbf{Z}
$$


in which $[L]$ is such that $\left[R_{\widetilde{T}}\right]=[L]^{T}[L]$. Further, $\mathbf{Z}=\left(\mathbf{Z}_{1}, \ldots \mathbf{Z}_{n_{t}}\right)$ is a Gaussian random vector such that $\mathcal{E}\left\{\mathbf{Z}_{j}\right\}=0, \mathcal{E}\left\{\mathbf{Z}_{j}^{2}\right\}=1$ and $\mathbf{Z}_{1}, \ldots, \mathbf{Z}_{n_{t}}$ are statistically independent. Note that Eq.(38) allows a generator of independent realizations $\mathbf{Z}\left(\theta^{\prime}\right)$ of $\mathbf{Z}$ to be constructed.

\subsection{Stochastic nonlinear computational model with random uncertainties}

Let $\{\mathbf{U}(\mathbf{x}, t), x \in \Omega, t \geq 0\}$ be the $\mathbb{R}^{n}$-valued, non-stationary in time, second-order stochastic process, defined on the product of probability spaces $(\Theta, \mathcal{T}, \mathcal{P})$ and $\left(\Theta^{\prime}, \mathcal{T}^{\prime}, \mathcal{P}^{\prime}\right)$. For $t \geq 0$, for all $\theta$ in $\Theta$ and for all $\theta^{\prime}$ in $\Theta^{\prime}$, a realization of the stochastic response is represented by its approximation $\mathbf{U}^{N}\left(\mathbf{x}, t ; \theta, \theta^{\prime}\right)$ of order $N$ such that

$$
\mathbf{U}^{N}\left(\mathbf{x}, t ; \theta, \theta^{\prime}\right)=\sum_{\beta=1}^{N} \boldsymbol{\varphi}^{\beta}(\mathbf{x}) \mathbf{Q}_{\beta}\left(t ; \theta, \theta^{\prime}\right)
$$

in which $\mathbf{Q}\left(t ; \theta, \theta^{\prime}\right)=\left(\mathbf{Q}_{1}\left(t ; \theta, \theta^{\prime}\right), \cdots, \mathbf{Q}_{N}\left(t ; \theta, \theta^{\prime}\right)\right)$ is the solution of the following nonlinear differential equation

$$
\begin{array}{r}
\mathcal{M}_{\alpha \beta}(\theta) \ddot{\mathbf{Q}}_{\beta}\left(t ; \theta, \theta^{\prime}\right)+\mathcal{D}_{\alpha \beta}(\theta) \dot{\mathbf{Q}}_{\beta}\left(t ; \theta, \theta^{\prime}\right) \\
\mathcal{K}_{\alpha \beta}^{(1)}(\theta) \mathbf{Q}_{\beta}\left(t ; \theta, \theta^{\prime}\right)+\mathcal{K}_{\alpha \beta \gamma}^{(2)}(\theta) \mathbf{Q}_{\beta}\left(t ; \theta, \theta^{\prime}\right) \mathbf{Q}_{\gamma}\left(t ; \theta, \theta^{\prime}\right) \\
\mathcal{K}_{\alpha \beta \gamma \delta}^{(3)}(\theta) \mathbf{Q}_{\beta}\left(t ; \theta, \theta^{\prime}\right) \mathbf{Q}_{\gamma}\left(t ; \theta, \theta^{\prime}\right) \mathbf{Q}_{\delta}\left(t ; \theta, \theta^{\prime}\right)=\mathcal{F}_{\alpha}\left(t ; \theta, \theta^{\prime}\right),
\end{array}
$$

with initial conditions

$$
\mathbf{Q}\left(0 ; \theta, \theta^{\prime}\right)=\mathbf{0} \quad, \quad \dot{\mathbf{Q}}\left(0 ; \theta, \theta^{\prime}\right)=\mathbf{0} .
$$

In Eq.(40), the quadratic stiffness term is written as

$$
\mathcal{K}_{\alpha \beta \gamma}^{(2)}(\theta)=\frac{1}{2}\left(\widehat{\mathcal{K}}_{\alpha \beta \gamma}^{(2)}(\theta)+\widehat{\mathcal{K}}_{\beta \gamma \alpha}^{(2)}(\theta)+\widehat{\mathcal{K}}_{\gamma \alpha \beta}^{(2)}(\theta)\right)
$$

Note that the random linear, quadratic and cubic stiffness terms $\mathcal{K}_{\alpha \beta}^{(1)}, \widehat{\mathcal{K}}_{\alpha \beta \gamma}^{(2)}$ and $\mathcal{K}_{\alpha \beta \gamma \delta}^{(3)}$ are easily deduced from the random matrix $[\mathcal{K}]$ using reshaped operations such as the one used in Eqs.(28)-(29). 


\subsection{Numerical aspects}

With regard to the numerical solver used for computing the nonlinear coupled differential equation, a Newmark method is used [39], which employs the averaging acceleration scheme known to be unconditionally stable. With this solver, a set of nonlinear algebraic equations must be solved at each sampling time. Such computation is addressed by the fixed-point method or by the Crisfield arc-length method [40]. The fixed-point method is favored because the iterative scheme does not require the evaluation of the tangential stiffness matrix. Nevertheless, when the algorithm does not converge, it is replaced by the Crisfield arc-length method. This latter algorithm introduces an additional scalar parameter that multiplies the right-hand member of the nonlinear equation and that has to be controlled. The iterative scheme requires the construction of the tangential stiffness at each iteration corresponding to a computed value of this additional scalar parameter. Contrary to the usual Newton-Raphson scheme, the main advantage of the procedure is its capability of capturing high non-linear complex mechanical behaviors including sudden softening and hardening of the response.

\section{Static post-buckling analysis of a cylindrical shell and experi- mental validation}

This Section is devoted to the experimental validation of the methodology on a thin cylindrical shell for which experimental data is available[8, 9]. The shell is modeled here using three-dimensional solid finite elements. In the present context, despite the very small thickness of the investigated structure, the choice of 3D solid finite elements is preferred to the more natural choice of shell finite elements for the following reasons:

- It is more exact to use a 3D nonlinear elasticity theory than a thin shell nonlinear elasticity theory.

- In the context of the construction of a reduced-order model, it is easier to compute the integrals defined by Eqs.(20) to (25).

- Finally, the computational cost increase, associated with the larger number of DOFs, is not a real obstacle, given the current computational capabilities and the possibility of using parallel computations. 
Experimental nonlinear static responses are available as a function of the static shear load magnitude[8, 9]. For comparison with this data, the stochastic computational model is constructed without the mass and the dissipation contributions. Furthermore, the external loading is deterministic.

\subsection{Description of the experiments}

The experimental data used in this paper is briefly summarized below, see [41] for complete details. The geometry of the cylindrical shell is characterized by the mean radius $R=0.125 \mathrm{~m}$, thickness $e=27010^{-6} \mathrm{~m}$ and height of $0.125 \mathrm{~m}$. The structure is described in a global cartesian coordinate system $\left(0, \mathbf{e}_{1}, \mathbf{e}_{1}, \mathbf{e}_{3}\right)$, where $O$ is the center of the ring basis and where the cylinder axis is defined along $\mathbf{e}_{3}$. It is composed of nickel, which is assumed to be a linear elastic material in the range of stresses considered. An electroplating process has been used for manufacturing the shell to obtain the most uniform thickness possible. This near uniformity allows the sensitivity of the post-buckling behavior with respect to geometric imperfections to be reduced. The bottom of the cylindrical shell is clamped to a base plate, assumed to be rigid. The top of the cylindrical shell is connected to a rigid plate, of thickness $0.019 \mathrm{~m}$, yielding a total height of the structure $h=0.144 \mathrm{~m}$. The loading is transmitted from servo-cylinders through this rigid plate. A constant traction load $F^{t}=8500 \mathrm{~N}$ is applied to delay the onset of the postbuckling. A controlled static shear point load $F^{s h}$ with maximum magnitude of $9750 \mathrm{~N}$, is then applied at the top of the shell along the direction $\mathbf{e}_{2}$. Let $\left(s_{1}, \cdots, s_{53}\right)$ be the sampling of the increasing static shear load, in which $s_{1}$ corresponds to $0 N$ and $s_{53}$ to $9750 N$. The displacement corresponding to a given static shear load increment $s_{j}$ is measured along the direction $\mathbf{e}_{2}$ at the observation point, which is the point at which the external load is applied. This measured displacement is denoted by $u_{\text {exp }}\left(s_{j}\right)$. It should be noted that only one load-displacement curve is available in the experimental data [41], see Fig. 1. Two different mechanical behaviors can be observed : (1) an elastic domain until the critical shear load $F_{\text {crit }}^{s h}=7450 N\left(s_{\text {crit }}=0.745\right)$ at which the displacement is $u_{c r i t}^{e x p}=1.53 \times 10^{-4} \mathrm{~m}$; (2) an approximately linear elastic behaviour in the postbuckling domain investigated as expected from $[6]$.

\subsection{Description of the mean finite element model}

The three-dimensional structure is modeled by a circular cylindrical shell of height $h=0.144 \mathrm{~m}$, mean radius $0.125 \mathrm{~m}$ and thickness $2.7 \times 10^{-4} \mathrm{~m}$. Its 


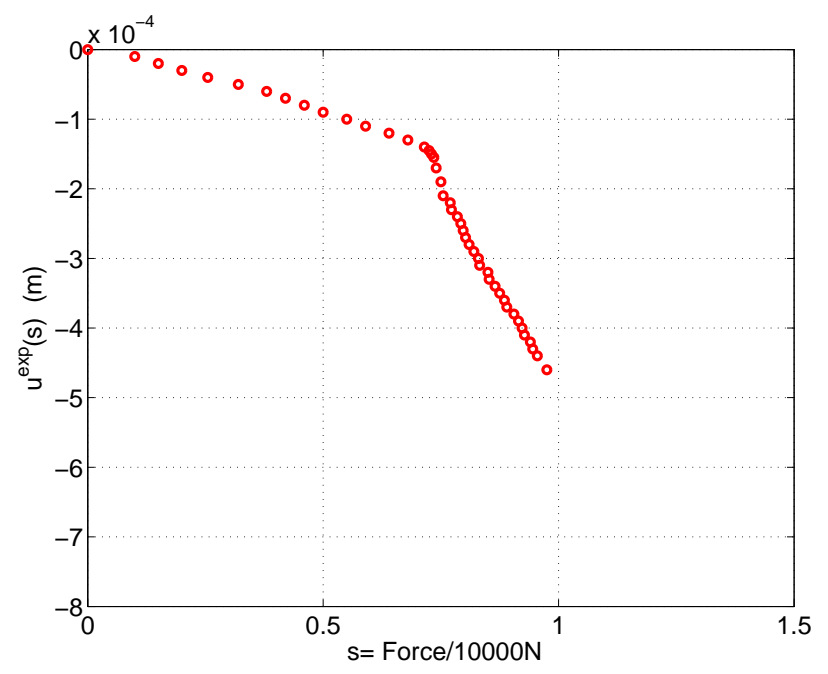

Figure 1. Experimental non-linear response $s \mapsto u^{\exp }(s)$

bottom is clamped and the upper ring is rigid with three DOFs in translation. The structure is subjected to the external shear point load $F^{s h}$ along $\mathbf{e}_{2}$ at $x_{3}=h$, and constant traction point load $F^{t}$ applied along $\mathbf{e}_{3}$ at $x_{3}=h$ - The isotropic linear elastic material properties have been experimentally measured to be $E=1.8 \times 10^{11} \mathrm{~N} . \mathrm{m}^{-2}$ and $\nu=0.3$. The mass density is taken as $\rho=8200 \mathrm{Kg} \times \mathrm{m}^{-3}$. It should be noted that a concentrated mass $M^{\prime}=80 \mathrm{Kg}$ is added at the free node located at the top of the rigid plate along direction $\mathbf{e}_{2}$. However, replacing the external traction load $F^{t}$ by $F^{\prime} t=$ $F^{t}+800 N$ does not change notably the experimental conditions described in the subsection above. Note that the impact of such mass modeling is negligible when dealing with the nonlinear static case but is required for the considered nonlinear dynamical case (see Section 5.2). The finite element model is a regular mesh composed of $\left(n_{r}-1\right) \times n_{\theta} \times\left(n_{3}-1\right)=1 \times 7500 \times$ $9599=7125008$-nodes solid finite elements with 8 Gauss integration points, in which $n_{r}, n_{\theta}$ and $n_{3}$ are the number of nodes along the radial, orthoradial and $\mathbf{e}_{3}$-axis directions. The mean computational model thus has 4230003 degrees of freedom. The observation is the displacement of the node obs, corresponding to the location of the experimental observation along $\mathbf{e}_{2}$ (the direction of the shear point load) and is denoted by $u_{o b s}(s)$.

The first linear elastic buckling mode, shown in Fig.2, was computed to 
occur for a critical shear load $F_{\text {crit }}=9400 N$. Although the experimental critical shear load is found to be $7450 \mathrm{~N}$, the computed buckling mode is found to be in agreement with its experimental counterpart [9]. A sensitivity analysis of the mean nonlinear computational model, is conducted in the linear elastic range with respect to the thickness $e$, the choice of boundary conditions and to the parameters $n_{\theta}$ and $n_{3}$ controlling the size of the mesh with the current 8-node finite elements. It is shown that the sensitivity is mainly due to the boundary conditions and that the set of chosen parameters yield these computational results to be the closest to the experimental ones.

It is known that the buckling load decreases with respect to initial imperfections $[6,7]$. In the present mean finite element model, a geometrical perturbation taken as the first linear buckling modal shape is added to the structure with a maximum amplitude of $2.7 \times 10^{-4} \mathrm{~m}$ corresponding to the thickness of the shell [9]. More generally, mainly due to the pattern of geometrical imperfections or to the boundary conditions, there is no reason for which the experimental post-buckling behavior of a given manufactured cylindrical shell would follow such branch. The available experimental data occurs according to this branch. Destroying slightly the axisymmetry of the structure according to this branch allows the possibility for the desired postbuckling branch to be captured but limits other post-buckling branches to be investigated.

\subsection{Static nonlinear analysis of the mean computational model}

The nonlinear static response of the finite element model will be referred to as the reference solution. As mentioned above, it should be underlined that the mean computational model has been voluntarily constructed with a chosen geometrical imperfection so that the features of the post-buckling branch explored by such mean computational model matches well the experimental post-buckling branch. Figure 3 shows the graph $s \mapsto u_{\text {obs }}(s)$ for $s$ belonging to $[0,1.5]$ which is to be compared with Fig.1. A quasi-linear elastic response can be observed for $s<0.45$ and then, a smooth transition corresponding to the buckling $(0.45<s<0.85)$. Seen next is a post-buckling with a quasilinear elastic behavior $(0.85<s<0.975)$ (similarly to Fig.1) until a linear elastic stiffening takes place $(0.975<s<1.5), s=1.5$ corresponds to a shear point load of $15000 \mathrm{~N}$ ). It should be noted that the smooth transition is directly related to the geometrical perturbation discussed above[9]. Figures 4 to 6 display the three-dimensional static deflections of the structure for 


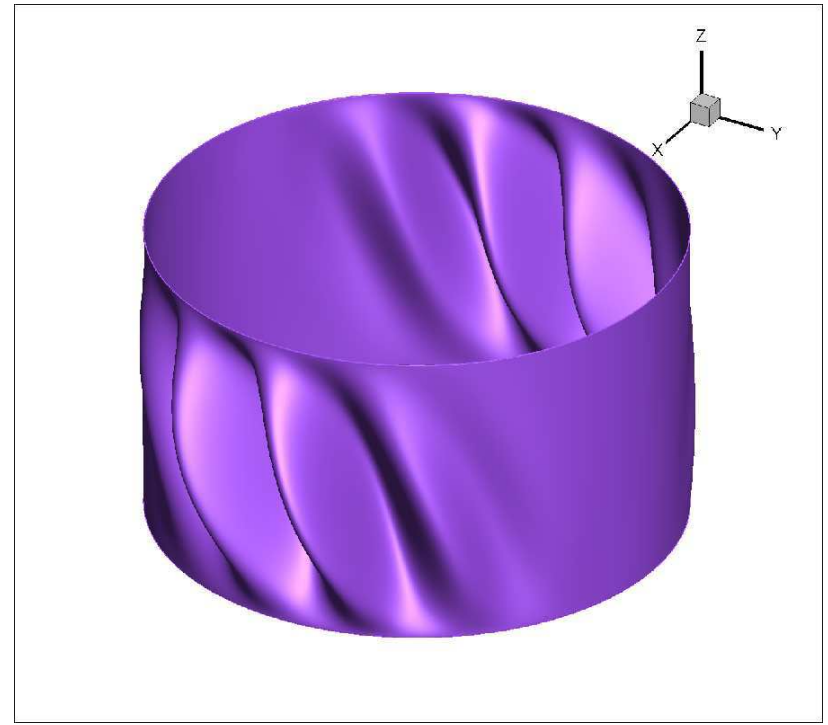

Figure 2. First computed linear elastic buckling mode

$s=0.45$ (quasi-linear elastic domain), $s=0.9$ (post-buckling quasi-linear elastic domain) and $s=1.25$ (elastic stiffening domain). 


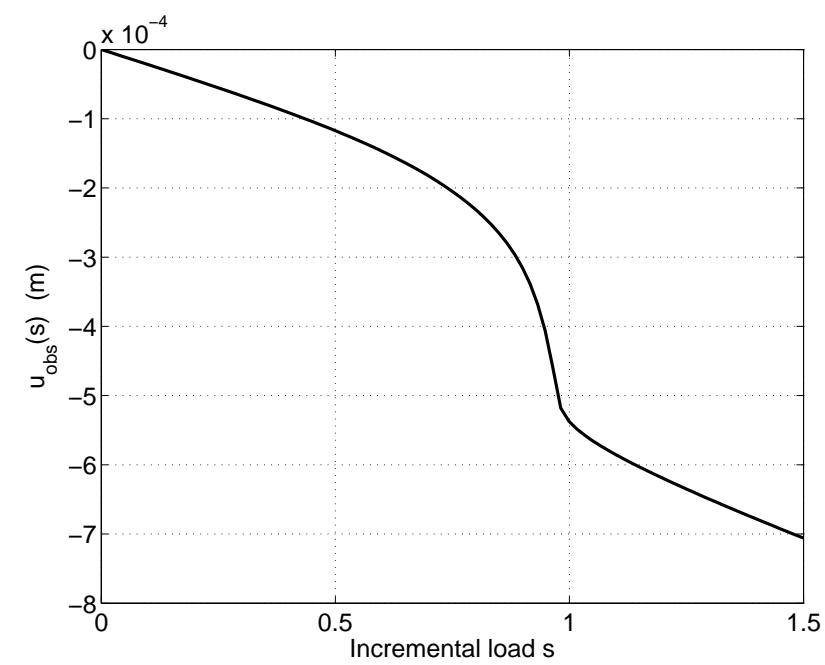

Figure 3. Nonlinear static response $s \mapsto u_{o b s}(s)$ obtained with the mean nonlinear computational model.

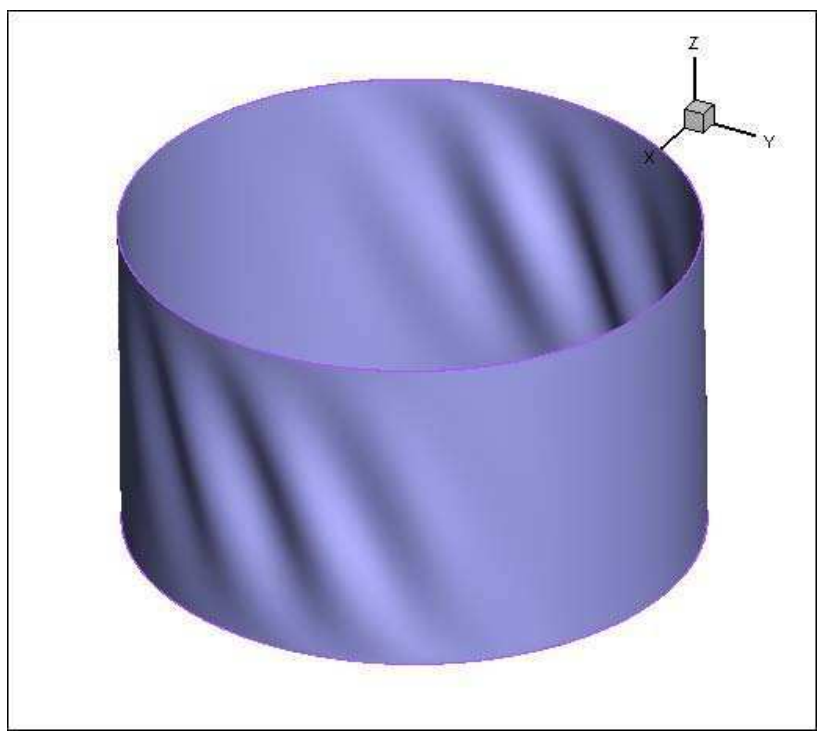

Figure 4. Displacement field of the nonlinear static response for the increment load $s=0.45$ with a zoom $(20 \times)$. 


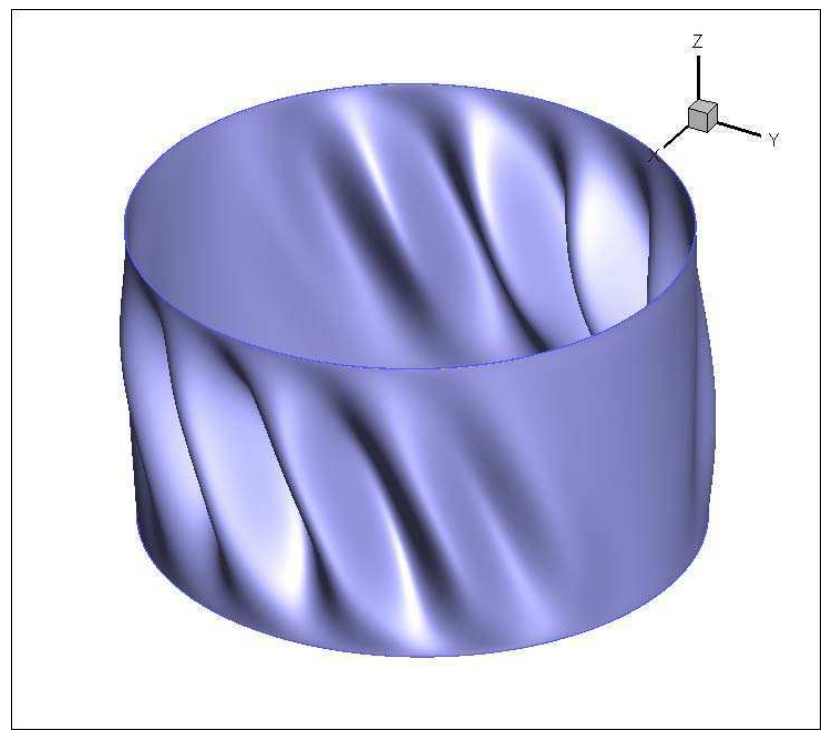

Figure 5. Displacement field of the nonlinear static response for the increment load $s=0.9$ with a zoom $(20 \times)$.

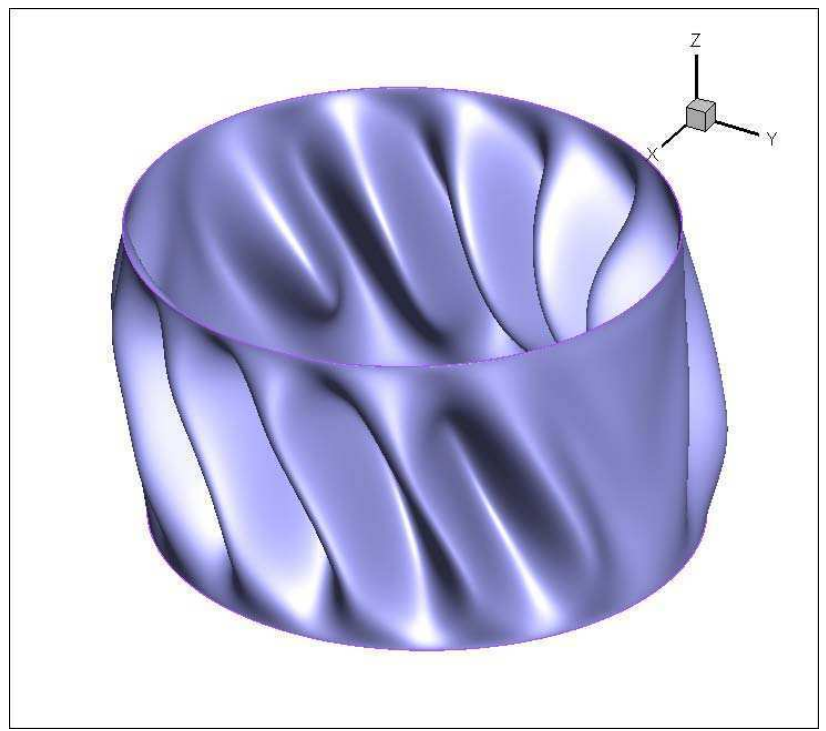

Figure 6. Displacement field of the nonlinear static response for the increment load $s=1.25$ with a zoom $(20 \times)$. 
4.4. Construction of the mean nonlinear reduced static computational model

The above nonlinear reference solution is then used for determining a projection basis with the Proper Orthogonal Decomposition method (PODmethod), which is known to be particularly relevant for nonlinear problems $[23,25,42,43,24,44]$.

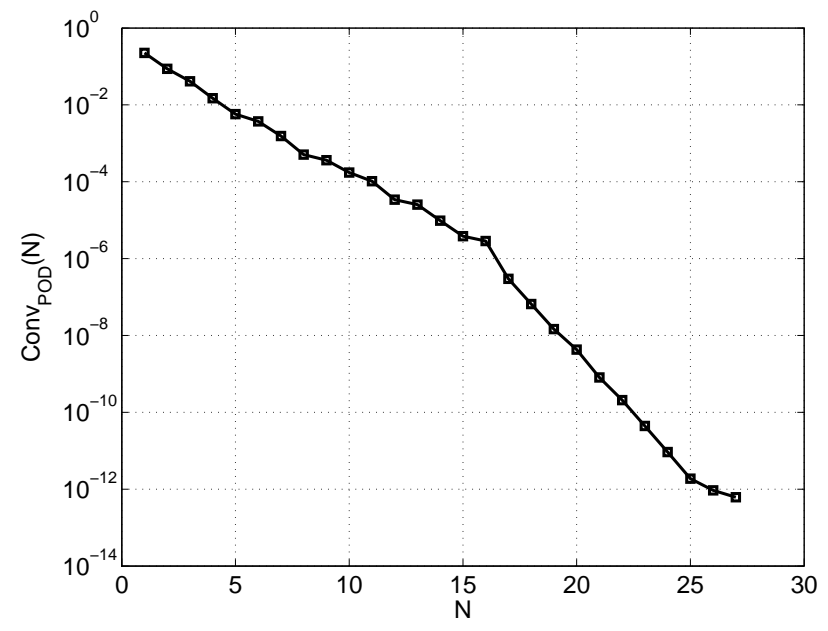

Figure 7. Convergence analysis : graph of $N \mapsto \operatorname{ConvPOD}_{\text {P }}(N)$.

Let $\mathbf{u}(s)$ be the $\mathbb{R}^{n}$ - displacement vector corresponding to the finite element discretization of the displacement field $\{\mathbf{u}(\mathbf{x}, s), \mathbf{x} \in \Omega\}$. In the present case, the POD method requires here to solve the eigenvalue problem for the correlation matrix related to the nonlinear static response $\{\mathbf{u}(s), s \in$ $[0,1.5]\}$. The eigenvectors $\varphi_{\alpha}$ (or POD modes), will form the vector basis of Eq.(18). Their corresponding eigenvalues $\lambda_{\alpha}$ are ordered by decreasing values. Note that this basis does not only depend on the operators of the mean nonlinear computational model but also strongly on the external loads applied to the structure. The numerical construction of the correlation matrix is especially difficult to achieve when dealing with large computational models. In such case, there also exist adapted strategies which allow the basis to be computed [23]. Assuming that the decreasing rate of the eigenvalues is sufficiently large, the basis is computed using the economy-size Singular Value Decomposition (SVD) algorithm [45] as follows. Let $s_{j}, j \in\{1, \ldots, p\}$ with $s_{j}<s_{j+1}$ be the scalar denoting the incremental weight number $j$ of 
the external load vector. Further, denote by $[V]$ the $(n \times p)$ real matrix

$$
\begin{aligned}
& {[V]_{i j}=u_{i}\left(s_{j}\right) \sqrt{\Delta s_{j}} \quad, \quad \Delta s_{j}=s_{j}-s_{j-1}} \\
& \text { with } s_{0}=0 .
\end{aligned}
$$

Therefore, the correlation matrix is written as

$$
[A]=[V][V]^{T}
$$

For fixed $N \leq n$ (note that in practice $N \lll n$ ), the economy-size SVD of matrix $[V]$ is written as

$$
[V]=\left[\Phi^{N}\right]\left[S^{N}\right]\left[C^{N}\right]^{T},
$$

where $\left[C^{N}\right]$ and $\left[\Phi^{N}\right]$ are such that $\left[C^{N}\right]^{T}\left[C^{N}\right]=\left[I_{N}\right]$ and $\left[\Phi^{N}\right]^{T}\left[\Phi^{N}\right]=$ $\left[I_{N}\right]$. Moreover, $\left[S^{N}\right]$ is a real positive $(N \times N)$ diagonal matrix such that $\left[S^{N}\right]_{\alpha \alpha}=\sqrt{\lambda_{\alpha}}$ with $\lambda_{1} \geq \lambda_{2} \geq \ldots \geq \lambda_{N}$. Finally, the columns of the $(n \times N)$ matrix $\left[\Phi^{N}\right]$, denoted by $\left[\varphi^{1}, \varphi^{2}, \cdots, \varphi^{N}\right]$, constitute the basis exhibiting the following orthonormal properties

$$
\left(\varphi^{\alpha}\right)^{T} \varphi^{\beta}=\delta_{\alpha \beta}
$$

A convergence analysis is carried out in order to select the number of POD modes which have to be kept in the numerical simulation.

Let $N \mapsto \operatorname{Conv}_{\mathrm{POD}}(N)$ be the relative error function defined as

$$
\operatorname{Conv}_{\mathrm{POD}}(N)=1-\frac{1}{\operatorname{tr}([A])} \sum_{j=1}^{N} \lambda_{j} .
$$

In regards to the evolution of the relative error, note that the computation of $\operatorname{tr}([A])$ does not require the explicit construction of matrix $[A]$ by using Eq. (44). Figure 7 shows the graph of $N \mapsto \operatorname{Conv}_{\mathrm{POD}}(N)$ which characterizes the convergence rate of the representation. It is seen that a good convergence is obtained for $N=27$, for which the relative error of Eq.(47) is close to numerical zero.

\subsection{Identification of the stochastic nonlinear static computational model}

Since the thin cylindrical shell is sensitive to small perturbations of its parameters (boundary conditions, ...), a compromise was necessary in the 
sensitivity analysis mentioned above, between improving the fit of the response in the quasi-linear elastic domain or in the post-buckling domain (controlled by the buckling load). A comparison of Figs. 1 and 3 confirms that the nonlinear computational model has the capability to reasonably represent the experimental response. Nevertheless, it remains sufficiently distant from the experiments to justify the implementation of uncertainties leading to a stochastic nonlinear computational model which will be identified using the experimental data. By construction, it should be noted that the present stochastic nonlinear computational model has the ability to quantify the scatter of the nonlinear response around the observed post-buckling direction. More precisely, the stochastic nonlinear computational model has the capability to a priori explore all the existing post-buckling branches. In the present case and by construction, the stochastic family explored by the computational model favors the post-buckling branch corresponding to the experimental branch of interest. Note that a few realizations corresponding to different post-buckling behaviors have been observed during the Monte Carlo simulation. Since such realizations do not correspond to the observed experimental branch, these realizations have not been considered in the statistics. For information purpose, $99.9 \%$ (or 98.7\%) of the 10000 realizations corresponding to the dispersion level $\delta=0.15$ (or $\delta=0.45$ ) belong to the post-buckling branch of interest and were used for estimating the Likelihood function.

The quasi-linear part of the elastic response constructed with the computational model yields acceptable results. The largest discrepancies between computational model and experimental predictions occur in the buckling and post-buckling regimes, it is in these loading conditions that the identification will be performed. This focus is consistent with the physical expectations that the response is most sensitive to uncertainties in these regimes. Based on the above discussion, these observations will be the experimented responses in the interval $s \in[0.75,0.975]$ which is sampled in 34 points denoted by $s_{i}, i=20 \ldots, 53$.

The construction of the stochastic nonlinear reduced-order computational model using the nonparametric probabilistic approach is performed as explained in Section 3.1. Let $U_{o b s}(s, \delta)$ be the random observations with which the parameter $\delta$ will be identified. There exists several methodologies adapted to the identification of stochastic computational models, see for instance $[46,47,48]$. In the present research, the identification procedure is achieved using the maximum likelihood method associated with a statistical reduction 


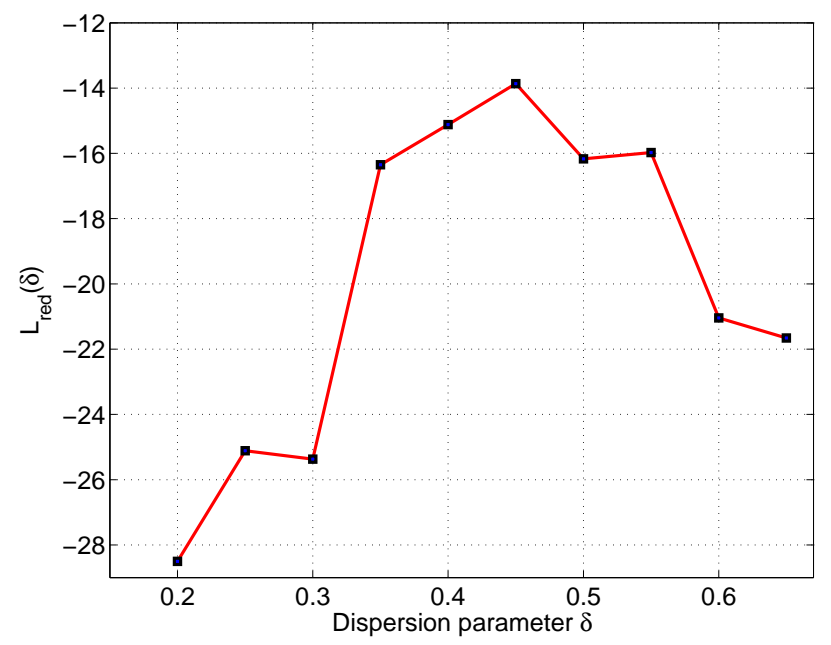

Figure 8. Graph of the likelihood function $\delta \mapsto \mathcal{L}^{\text {red }}(\delta)$.

of the information [49]. Note that it is particularly adapted to the present case for which the random variables $U_{\text {obs }}\left(s_{i}, \delta\right)$ are not only dependent but also strongly correlated. This approach proceeds with a principal component analysis of the random observations which leads to a reduced set of uncorrelated random variables. The likelihood function, $\delta \mapsto \mathcal{L}^{\text {red }}(\delta)$, is evaluated in the space of these uncorrelated random variables representing the data. It is then obtained with a reduction to 5 uncorrelated random variables and for $\delta \in \Delta=[0.2,0.65]$. It was found that the use of 5 uncorrelated variables yields a relative error of $5.41 \%$ on the trace of the covariance matrix, which was deemed to be sufficiently small. Figure 8 displaying the graph of the likelihood function $\delta \mapsto \mathcal{L}^{r e d}(\delta)$, was obtained using the Monte Carlo numerical simulation with $n_{s}=10000$ independent realizations. It has been observed through a convergence analysis that 4000 independent realizations yields a good convergence. Note that the likelihood function is replaced by an approximation which is constructed as the product of marginal probability density functions of each uncorrelated random variable. Although this assumption modifies the Likelihood function, it has been shown that its combination with the statistical reduction yield accurate estimations [49]. Seeking the maximum of $\mathcal{L}^{\text {red }}(\delta)$ for the experimental identification yields the optimal value $\delta^{\text {opt }}=0.45$. 


\subsection{Confidence region analysis}

The confidence region of the static nonlinear response predicted with the stochastic nonlinear reduced-order model is then computed with a probability level $P_{c}=0.95$ using the quantile method [50], see Figure 9.

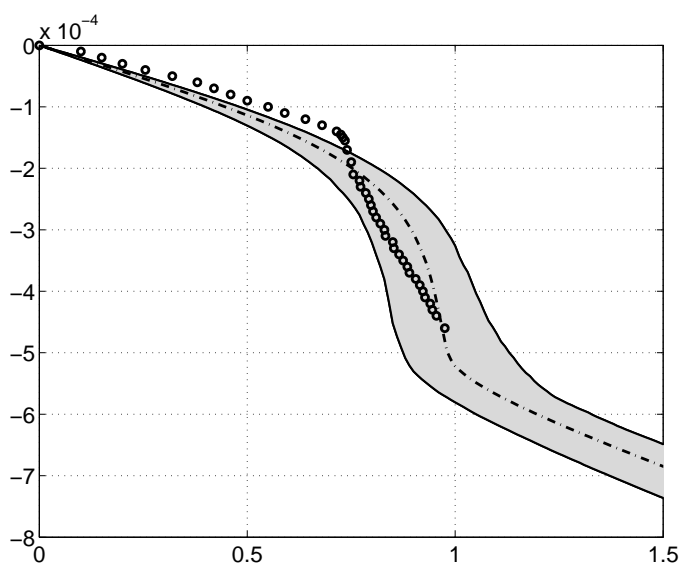

Figure 9. Experimental identification : graph of experimental data $s \mapsto u^{\exp }(s)(o$ markers), graph of the reference response $s \mapsto u^{o b s}(s)$ (thick dashed line), graph of the confidence region of the random response $s \mapsto U_{o b s}(s, \delta=0.45)$ (grey region).

Also shown on this figure is the experimental nonlinear response. Despite the slight underestimation provided by the optimal stochastic nonlinear computational model in the linear range (induced by the choice of the mean nonlinear computational model as explained above), the results are broadly consistent with the experimental nonlinear response, validating the stochastic nonlinear reduced-order model and its identification.

Analyzing the features of the confidence region shown in Fig. 9, it is first observed that the effects of uncertainties increase with the nonlinearity as may be expected. In the buckling and post-buckling range, for which the static load increment $s$ belongs to $[0.45,0.975]$, the confidence region quickly broadens, allowing some realizations of the random displacement to be almost doubled of others. Consider next the elastic stiffening range, corresponding to a static load increment $s>0.975$, for which no experimental result is available but which can be predicted by the stochastic nonlinear computational model. First, it is seen that the mechanical behavior tends to be a linear one. Moreover, the confidence region is more narrow in this range than in the previous one, which demonstrates a robustness of the random 
response with respect to both model and system-parameter uncertainties. Figure 10 to 12 show the mean of the static response (deflection) and its standard deviation (colormap) for static load increments $s=0.45, s=0.9$, $s=1.3$. Again, it is seen that uncertainty increases during the buckling and post-buckling phases, and decreases at higher deflection levels corresponding to the elastic stiffening range.

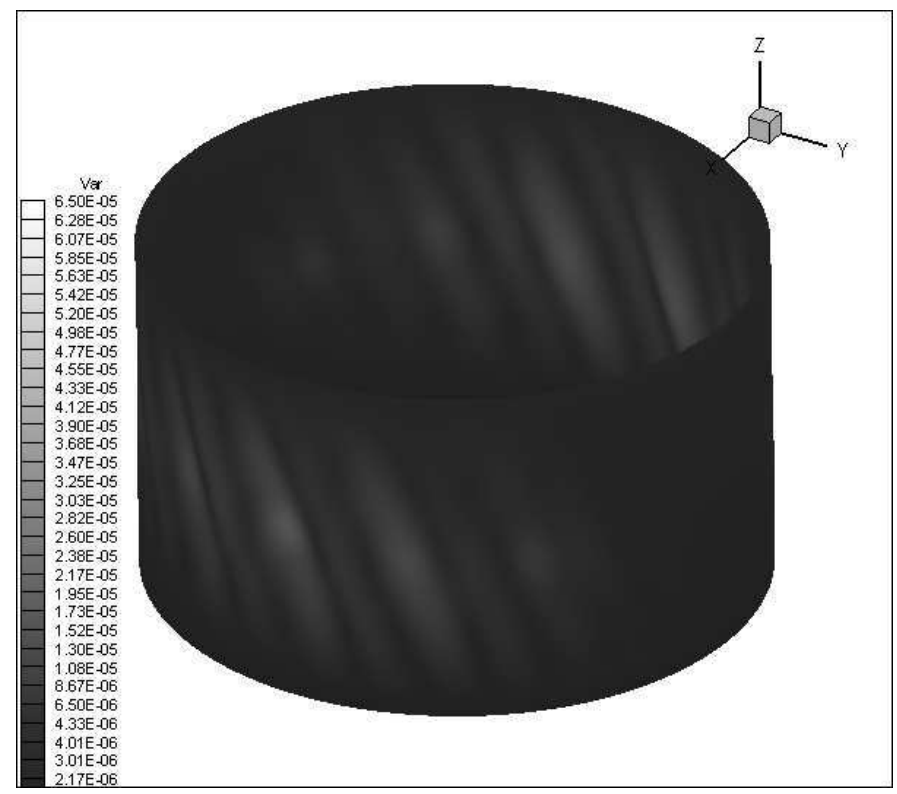

Figure 10. Uncertainty propagation : deflection and standard deviation (colormap) of the cylindrical shell for an incremental shear load $s=0.45$ 


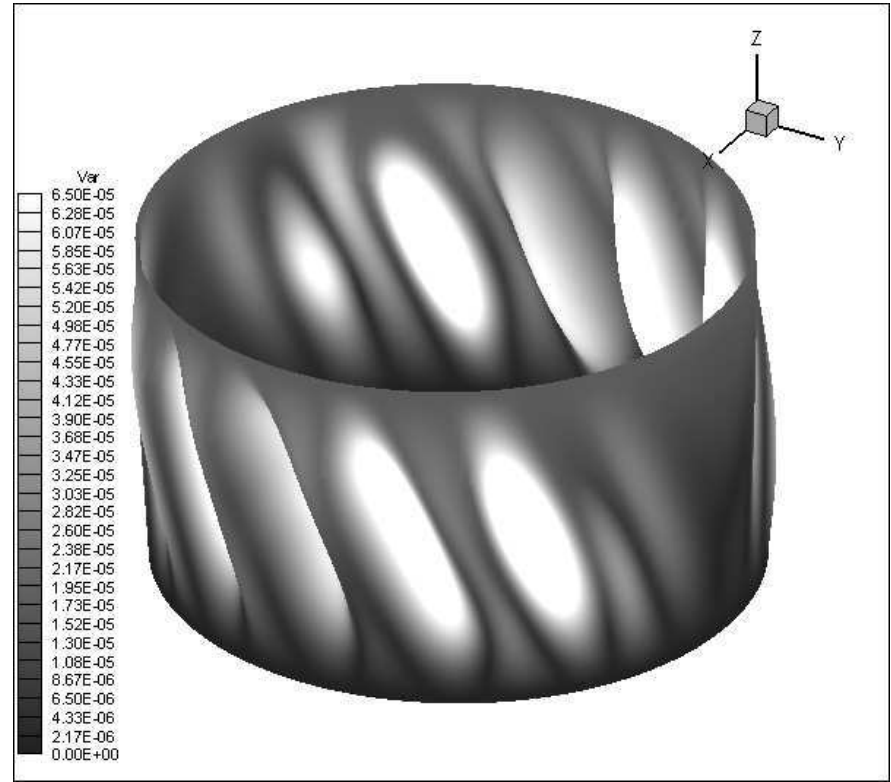

Figure 11. Uncertainty propagation : deflection and standard deviation (colormap) of the cylindrical shell for an incremental shear load $s=0.9$ 


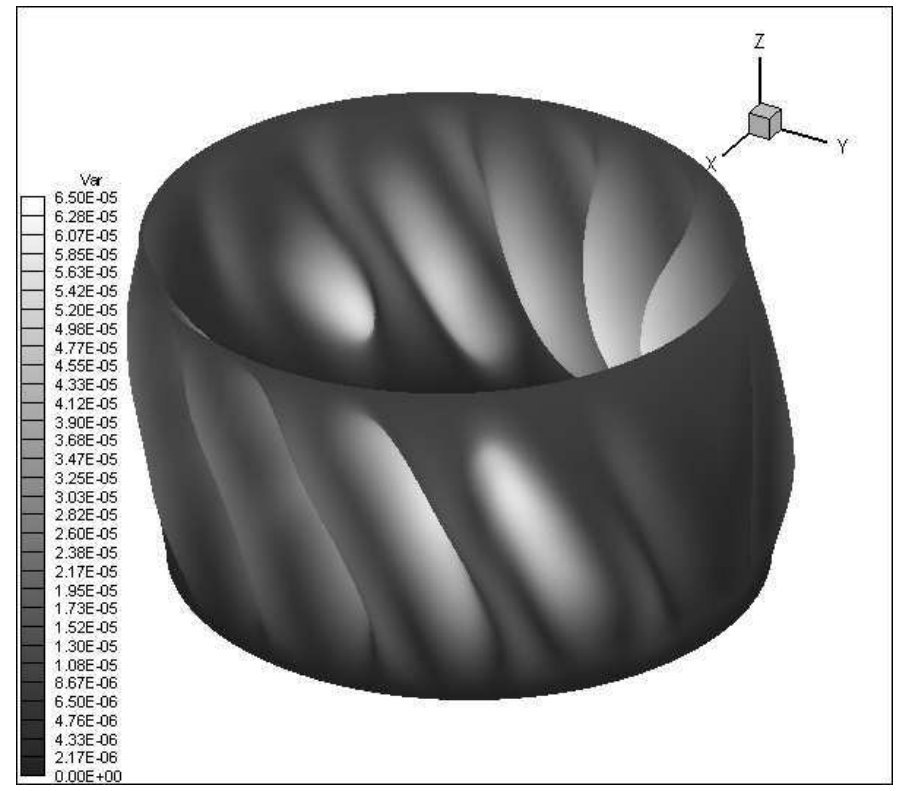

Figure 12. Uncertainty propagation : deflection and standard deviation (colormap) of the cylindrical shell for an incremental shear load $s=1.3$ 


\section{Dynamic post-buckling analysis of the cylindrical shell under a seismic ground motion}

This section concerns the dynamical analysis of the cylindrical shell subjected to an earthquake based-ground motion. The stochastic nonlinear computational model of the shell constructed and identified in the previous Section is reconvened with $\delta_{K}=\delta_{K}^{o p t}=0.45$. Its random operators are defined on the probability space $(\Theta, \mathcal{T}, \mathcal{P})$. Uncertainties on the mass and damping are neglected here but could easily be introduced. The dynamic loading conditions consist in a seismic horizontal motion, see Section 3.2, applied to the base of the cylindrical shell. The mass being deterministic, the corresponding shear load is a stochastic process indexed by $\mathbb{R}^{+}$and defined on the probability space $\left(\Theta^{\prime}, \mathcal{T}^{\prime}, \mathcal{P}^{\prime}\right)$. Consequently, the stochastic physical response is modeled by $\mathbb{R}^{n}$-random nonlinear stochastic process $\left\{\mathbf{U}(t), t \in \mathbb{R}^{+}\right\}$defined on the product of probability spaces $(\Theta, \mathcal{T}, \mathcal{P})$ and $\left(\Theta^{\prime}, \mathcal{T}^{\prime}, \mathcal{P}^{\prime}\right)$.

\subsection{Definition of the stochastic excitation and of the frequency band of anal- ysis}

The stochastic excitation is simulated as explained in Section 3.2. For the present application, the power spectral density $S_{\beta}(\omega)$ of the stochastic process $\boldsymbol{\beta}(t)$ is chosen as

$$
S_{\beta}(\omega)=\bar{s} \frac{\bar{\omega}^{4}+4 \bar{\xi}^{2} \bar{\omega}^{2} \omega^{2}}{\left(\omega^{2}-\bar{\omega}^{2}\right)^{2}+4 \bar{\xi}^{2} \bar{\omega}^{2} \omega^{2}},
$$

in which $\bar{s}=1.15 \times 10^{-4} \mathrm{~m}^{2} . \mathrm{s}^{-3}, \bar{\omega}=785.4 \mathrm{rad} . \mathrm{s}^{-1}$ and $\bar{\xi}=0.02$. The frequency band of analysis is $\mathbb{B}_{\nu}=[0,160] \mathrm{Hz}$. The time sampling is $\delta t=$ $5 \times 10^{-4} \mathrm{~s}$, the total time duration $T=1.27 \mathrm{~s}$ and therefore, the number of time steps is $n_{t}=2546$. The frequency resolution is $\delta \nu=0.78 \mathrm{~Hz}$ corresponding to a frequency band $[0,1000] \mathrm{Hz}$ (the sampling frequency is $\left.\nu_{e}=2000 \mathrm{~Hz}\right)$. The envelope function is a piecewise continuous function defined by $g(t)=25 t^{2} / 4$ if $t<0.4 \mathrm{~s}, g(t)=1$ if $t \in[0.4,0.7] \mathrm{s}$ and $g(t)=e^{-10(t-0.7)}$ if $t>0.7 \mathrm{~s}$. Figure 13 displays a typical realization $\boldsymbol{\Gamma}\left(\theta^{\prime}, t\right)$ of the stochastic process $\boldsymbol{\Gamma}(t)$. The stochastic excitation is then obtained using Eq.(34).

\subsection{Construction of the mean nonlinear reduced-order computational model}

For the considered nonlinear elasto-dynamic problem, there are two notable strategies for constructing the projection basis of the mean nonlinear 


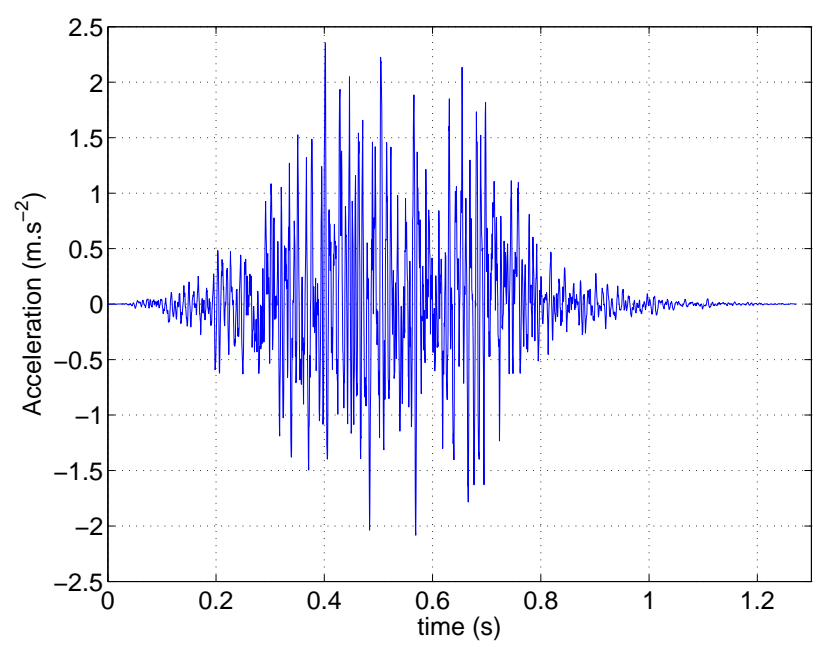

Figure 13. Realization of stochastic process $\boldsymbol{\Gamma}(t)$

reduced-order computational model. A first one uses the POD method and thus requires full computations of the elasto-dynamical reference response with the nonlinear computational model. A second strategy consists in solving the usual generalized eigenvalue problem of the linearized computational model and selecting a set of its linear eigenmodes of vibration as projection basis. Once this basis is built according to either of the above strategies, the mean nonlinear reduced operators are constructed by the methodology of Section 2.

It would be interesting to reuse the POD projection basis obtained in Section 4 for the present nonlinear dynamic case. Since the convergence rate of the solution constructed with such a projection basis strongly depends on the external applied loads, it is appropriate only if a similar loading class is used. With the presence of the concentrated mass $M^{\prime}$ at the top of the structure, the shear load induced by the ground motion is largest at the top of the structure. Its spatial shape is then close to the spatial repartition of the controlled shear load used for the nonlinear static case. Consequently, the dynamical excitation, in addition to the external constant traction load, is compatible with the loading used for the nonlinear static case and thus the projection basis of Section 4 can be used again. Specifically, let $\mathcal{R}^{(N)}=$ $\left\{\varphi^{1}, \ldots, \varphi^{N}\right\}$ be the family composed of the $N$ first basis vectors $\varphi^{1}, \ldots, \varphi^{N}$ of the static POD basis constructed in Section 4.4. Here $N=27$. Note that 
the family $\mathcal{R}^{(27)}$ is not expected to be sufficient to describe the nonlinear dynamical response of the shell; it needs to be completed by additional basis vectors that we select as linear elastic modes. A usual modal analysis of the linearized dynamical computational model with the predeformation discussed in Section 4.2 is then performed of which some results are summarized in Table 1.

\begin{tabular}{|c|c|c|}
\hline & frequency $(\mathrm{Hz})$ & modal shape \\
\hline Mode 1 & 121.10 & global \\
\hline Mode 2 & 124.16 & global \\
\hline Mode 3 & 897.02 & local \\
\hline Mode 4 & 897.06 & local \\
\hline
\end{tabular}

Table 1: Eigenfrequencies and type of elastic modes

Owing to the slight predeformation of the structure, according to the first linear buckling mode, the structure is not perfectly axisymmetric and pairs of distinct eigenfrequencies are obtained as seen in Table 1. An analysis of the first 40 eigenfrequencies and mode reveals that the first two modes $\tilde{\varphi}^{1}$ and $\tilde{\varphi}^{2}$ describe global bending modes of the structure as shown in figures 14 and 15 , see Table 1 .

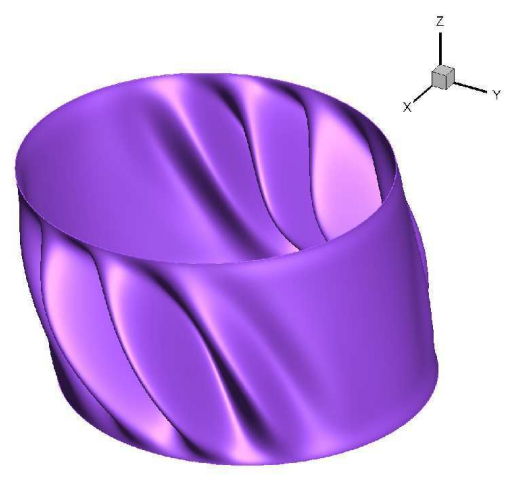

Figure 14. Elastic mode related to eigenfrequency $f_{1}=121.10 \mathrm{~Hz}$

Further, a high modal density is observed, starting with eigenfrequency $\nu_{3}=897.02 \mathrm{~Hz}$. There are 38 local elastic modes belonging to frequency 


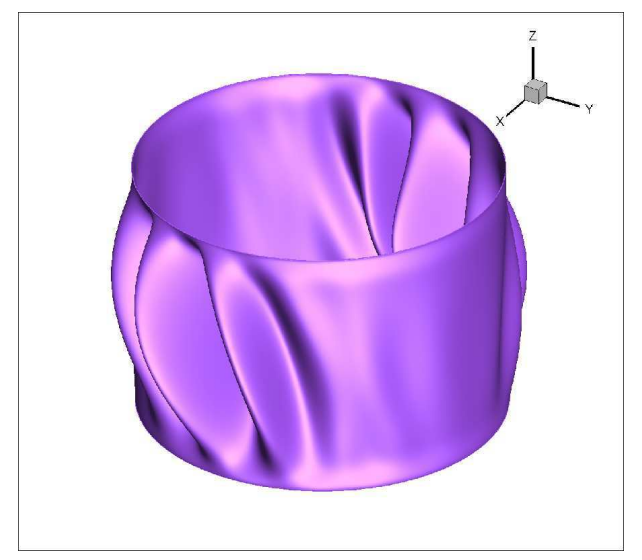

Figure 15. Elastic mode 2 related to eigenfrequency $f_{2}=124.16 \mathrm{~Hz}$

band $[897,1123] \mathrm{Hz}$. The ground motions characterized by the power spectral density of Eq.(48) would strongly excite the first two elastic modes but only weakly the ensuing ones. Thus the projection basis of the stochastic nonlinear reduced-order model must closely represent the first two elastic modes but not necessarily the ensuing ones. To assess this issue, the representation of elastic mode $\widetilde{\varphi}^{\beta}, \beta \in\{1,2\}$ on $\mathcal{R}^{(27)}$ is determined, using Eq.(46). Specifically, denote by $\widetilde{\varphi}^{\beta, N}$ the approximation of $\widetilde{\varphi}^{\beta}$ on $\mathcal{R}^{(N)}$ and let truncation error be given by

$$
\operatorname{Err}(N, \beta)=\frac{\left\|\widetilde{\varphi}^{\beta, N}-\widetilde{\varphi}^{\beta}\right\|}{\left\|\widetilde{\varphi}^{\beta}\right\|} .
$$

Figure 16 displays the graphs of $N \mapsto \operatorname{Err}(N, 1)$ and $N \mapsto \operatorname{Err}(N, 2)$ in a logarithmic scale. It is seen that elastic mode $\widetilde{\varphi}^{1}$ is poorly represented on $\mathcal{R}^{(27)}$. Although the error related to elastic mode $\tilde{\varphi}^{2}$ is smaller, due to the coexistence of local and global contributions, it was deemed too large to be neglected. As a consequence, these two elastic modes were selected in order to enrich $\mathcal{R}^{(27)}$. The final projection basis is the family $\mathcal{S}^{(29)}=$ $\mathcal{R}^{(27)} \bigcup\left\{\varphi^{28}, \varphi^{29}\right\}$, in which the vectors $\varphi^{\alpha}$, for $\alpha \in\{28,29\}$ are defined as

$$
\boldsymbol{\varphi}^{\alpha}=\frac{\boldsymbol{\psi}^{\alpha}}{\left\|\boldsymbol{\psi}^{\alpha}\right\|} \quad, \quad \boldsymbol{\psi}^{\alpha}=\widetilde{\boldsymbol{\varphi}}^{\alpha-27}-\sum_{i=1}^{\alpha-1} \frac{\left(\boldsymbol{\varphi}^{i}\right)^{T} \widetilde{\boldsymbol{\varphi}}^{\alpha-27}}{\left\|\boldsymbol{\varphi}^{i}\right\|^{2}} \boldsymbol{\varphi}^{i}
$$

according to a partial Gram-Schmidt orthonormalization procedure. The mean reduced-order computational model is then obtained using the en- 


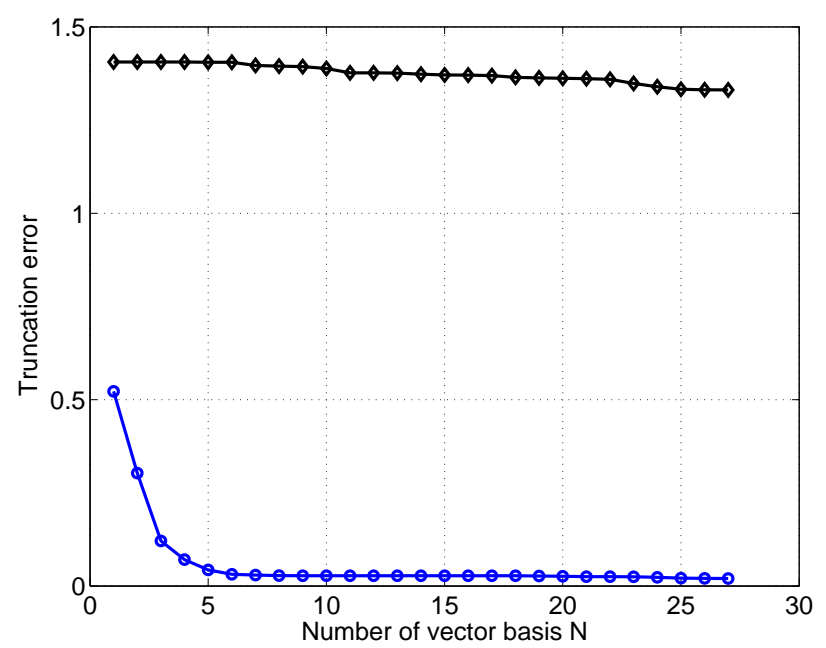

Figure 16. Graph of the error functions $N \mapsto \operatorname{Err}(N, 1)(\bullet$ symbol) and of $N \mapsto$ $\operatorname{Err}(N, 2)($ symbol $)$

riched projection basis composed by the family $\mathcal{S}^{(29)}$. It should be noted that the first eigenfrequency is computed with the linearized computational model projected on $\mathcal{S}^{(29)}$, are $\nu_{1}^{\prime}=121.19 \mathrm{~Hz}<\nu_{2}^{\prime}=124.57 \mathrm{~Hz}<\nu_{3}^{\prime}=$ $1602.96 \mathrm{~Hz}<\nu_{4}^{\prime}=1768.25 \mathrm{~Hz}$. As expected, $\nu_{1}^{\prime}$ and $\nu_{2}^{\prime}$ are close to their original counterparts $\nu_{1}$ and $\nu_{2}$, see Table 1 . The reduced dissipation matrix is constructed according to Eq.(26) by choosing $\zeta=4.44 \times 10^{-5}$. This leads to a critical dissipation rate $\xi \simeq 0.017$ for the two first elastic modes.

\subsection{Results}

The response of the shell is monitored at the same position and in the same direction than above, see Section 4.2. This random observation, denoted by $U_{o b s}(t)$, is a stochastic process indexed by $[0, T]$ and defined on the product of probability spaces $(\Theta, \mathcal{T}, \mathcal{P})$ and $\left(\Theta^{\prime}, \mathcal{T}^{\prime}, \mathcal{P}^{\prime}\right)$. It can then be written as

$$
U_{o b s}(t)=\underline{u}_{o b s}(t)+U_{o b s}^{c}(t),
$$

in which $\underline{u}_{o b s}(t)$ is the deterministic function characterizing the mean of the stochastic nonlinear dynamical response i.e.

$$
\underline{u}_{o b s}(t)=\mathcal{E}\left\{U_{o b s}(t)\right\}=\int_{\Theta} \int_{\Theta^{\prime}} U_{o b s}\left(t, \theta, \theta^{\prime}\right) d \mathcal{P}(\theta) d \mathcal{P}^{\prime}\left(\theta^{\prime}\right)
$$


and where $U_{o b s}^{c}(t)$ is a centered non-stationary stochastic process. In the sequel, $U_{o b s}\left(t, \theta, \theta^{\prime}\right)$ is denoted by $U_{o b s}(t ; \theta)$, where $\mathbb{\theta}=\left(\theta, \theta^{\prime}\right)$.

Four analysis cases (see Table 2 ) are investigated, analyzed and compared.

\begin{tabular}{|l|c|c|c|}
\hline & Stiffness & Stiffness & External load \\
\hline Case 1 & linear & deterministic & stochastic \\
\hline Case 2 & linear & stochastic & stochastic \\
\hline Case 3 & nonlinear & deterministic & stochastic \\
\hline Case 4 & nonlinear & stochastic & stochastic \\
\hline
\end{tabular}

Table 2: Description of the analysis cases

Figure 17 displays the graphs of $t \mapsto U_{o b s}^{c}(t, \theta)$ (gray line) and $t \mapsto \underline{u}_{o b s}(t)$ (black line) for the four analysis cases and for a specific realization of the ground motions related to Fig. 13. It is seen that $\underline{u}_{o b s}(t)$ is a centered oscillating function for the linear cases as expected given the zero mean character of the excitation. However, a small negative mean is observed for the nonlinear cases. Superimposing all cases, it is seen, for this present realization, that the geometric nonlinear effects occur first at $t_{\text {nonlin }}=0.16 \mathrm{~s}$ for a displacement level greater than $1.8 \times 10^{-4} \mathrm{~m}$, or $\frac{2}{3}$ of the shell thickness. Note that these observations are coherent with the results obtained in the static case and shown in Figure 3. Comparing cases 1 and 2 with cases 3 and 4 respectively, it is seen that the effect of the geometrical nonlinearities is to decrease the intensity of the response. Moreover, this effect increases with the presence of model and system-parameter uncertainties in the stochastic computational model. Comparing case 1 with case 2, it is seen that the presence of random uncertainties significantly spreads the stochastic linear response allowing displacements of $1.7 \times 10^{-3} \mathrm{~m}$, or 6 thicknesses, to occur. A similar conclusion does not seem to hold in the nonlinear case : when comparing case 3 with case 4 , the presence of random uncertainties only seems to slightly modify the nonlinear dynamical response.

The complex instantaneous spectral density function $s_{U_{o b s}^{c}}(\nu, t)$ of the nonstationary stochastic process $\left\{U_{o b s}^{c}(t), t \in[0, T]\right\}$ is defined as

$$
s_{U_{o b s}^{c}}(\nu, t)=\frac{1}{2 \pi} \int_{0}^{T} e^{-2 i \pi \nu t^{\prime}} r_{U_{o b s}^{c}}\left(t^{\prime}, t\right) d t^{\prime}
$$

in which $r_{U_{o b s}^{c}}\left(t, t^{\prime}\right)=\mathcal{E}\left\{U_{o b s}^{c}(t) U_{o b s}^{c}\left(t^{\prime}\right)\right\}$ is the autocorrelation function of stochastic process $U_{o b s}^{c}(t)$. It should be noted that the numerical estimation 

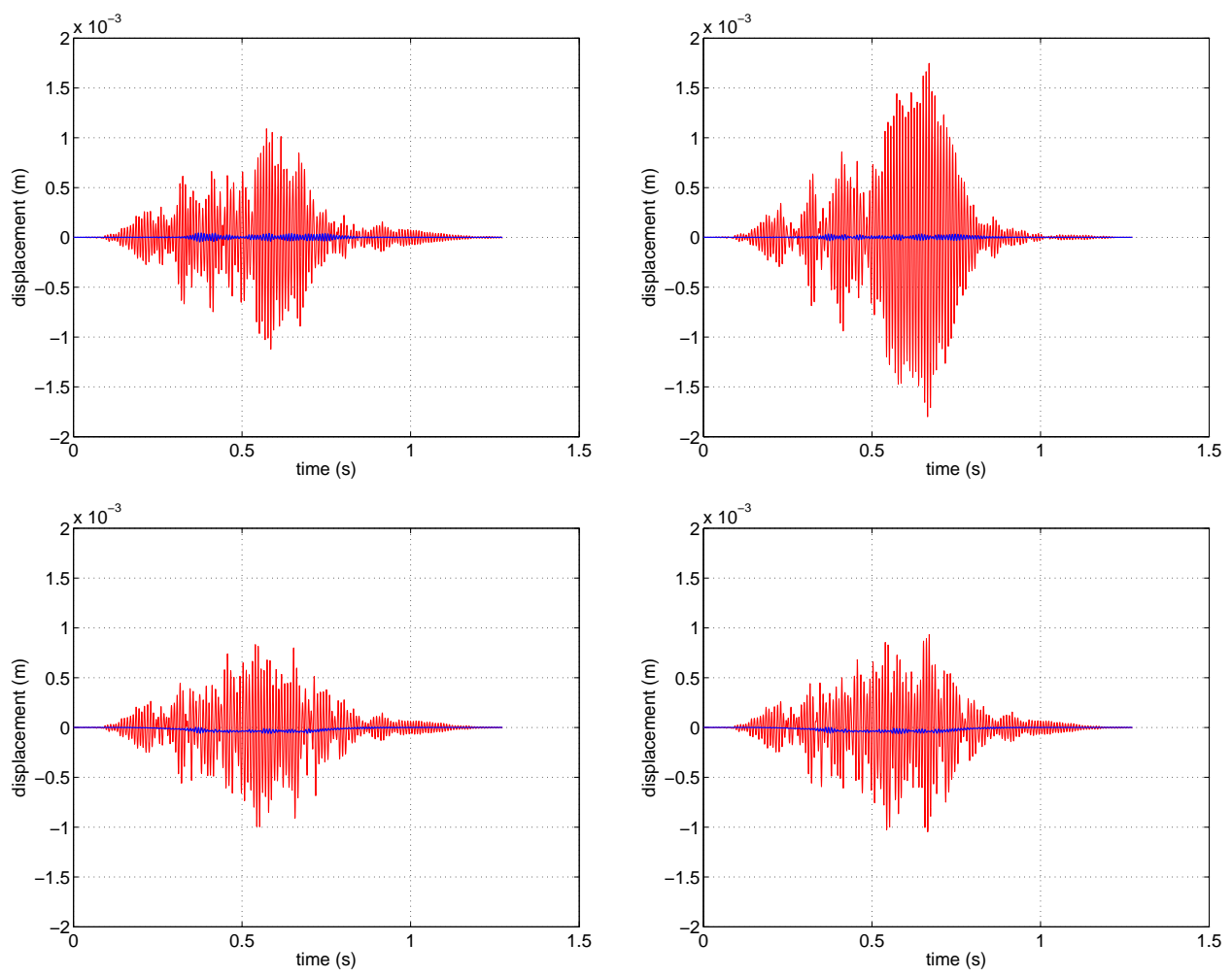

Figure 17. Graphs of functions $t \mapsto U_{o b s}^{c}(t, \theta)$ (gray line) and $t \mapsto \underline{u}_{o b s}(t)$ (black line) for cases 1 and 2 (upper graphs) and for cases 3 and 4 (lower graphs)

of $s_{U_{\text {obs }}^{c}}(\nu, t)$ can be carried out by using the periodogram method with a Tukey-Hanning time window combined to FFT $[51,52]$. Then,

$$
s_{U_{o b s}}(\nu, t)=\frac{1}{2 \pi} \underline{\hat{u}}_{o b s}(\nu) \underline{u}_{o b s}(t)+s_{U_{o b s}^{c}}(\nu, t),
$$

with $\underline{\hat{u}}_{o b s}(\nu)=\int_{0}^{T} e^{-2 i \pi \nu t^{\prime}} \underline{u}_{o b s}\left(t^{\prime}\right) d t^{\prime}$.

Figure 18 displays the graph $(\nu, t) \mapsto\left|s_{U_{o b s}}(\nu, t)\right|$ for the four analysis cases. It is seen that the two linear elastic modes are excited through the analysis, as expected from the eigenfrequencies $\nu_{i}^{\prime}$ computed in Section 5.2. The analysis is next focused on the fixed time $t_{0}=0.5 \mathrm{~T}$ and in the frequency band $[80,160] \mathrm{Hz}$ around the resonances. Figure 19 shows the graph $\nu \mapsto \log _{10}\left(\left|s_{U_{o b s}}\left(\nu, t_{0}\right)\right|\right)$ for the linear case (left graph) and for the nonlinear 
case (right graph). The presence of random uncertainties spreads the resonance in the linear case whereas no such effect is noticed in the nonlinear case. Comparing the peak frequencies of cases 1 and 3, a shift of the resonance from $120 \mathrm{~Hz}$ until $113 \mathrm{~Hz}$ is observed, which shows that the presence of geometrical nonlinearities induces a softening effect, as maybe expected from Fig. 3. Moreover, the presence of geometrical nonlinearities yields a peak broadening and a decrease of the resonance magnitude from $-8.3 d B$ until $-9.16 d B$. Comparing case 2 (or 4 ) with case 1 (or 3 ), it is seen that the presence of uncertainties have a moderate effect on the complex instantaneous spectral density function of the response. For the linear case, the presence of uncertainties shifts the peak from $120 \mathrm{~Hz}$ to $118 \mathrm{~Hz}$ and reduces its magnitude by $0.2 \mathrm{~dB}$. This small effect can be explained as follows: in case 2, the random linear $(N \times N)$ reduced stiffness operator is extracted from the $(P \times P)$ random reshaped stiffness operator $[\mathcal{K}]$, for which the dispersion parameter $\delta$ has been identified to 0.45 . Let $\delta^{L I N}$ be the effective dispersion parameter related to this random linear $(N \times N)$ reduced stiffness operator. Since $N \ll P=N(N+1)$, it can be shown that $\delta^{L I N}=0.025$. Consequently, the random response of the linearized system with uncertainties is computed with a small level $\left(\delta^{L I N}=0.025\right)$ of uncertainties. This small level of the dispersion parameter is then coherent with the observed slight impact of the random uncertainties on the linear dynamical response. In the nonlinear case, only a change of $0.03 \mathrm{~dB}$ can be observed in the peak response magnitude. For both nonlinear and linear cases, the complex instantaneous spectral density function appears weakly sensitive to random uncertainties. This can be explained by the fact that this quantity of interest is issued from a statistical averaging of the random observation over the two statistical sources of the randomness (the excitation and the variability induced by uncertainties). 

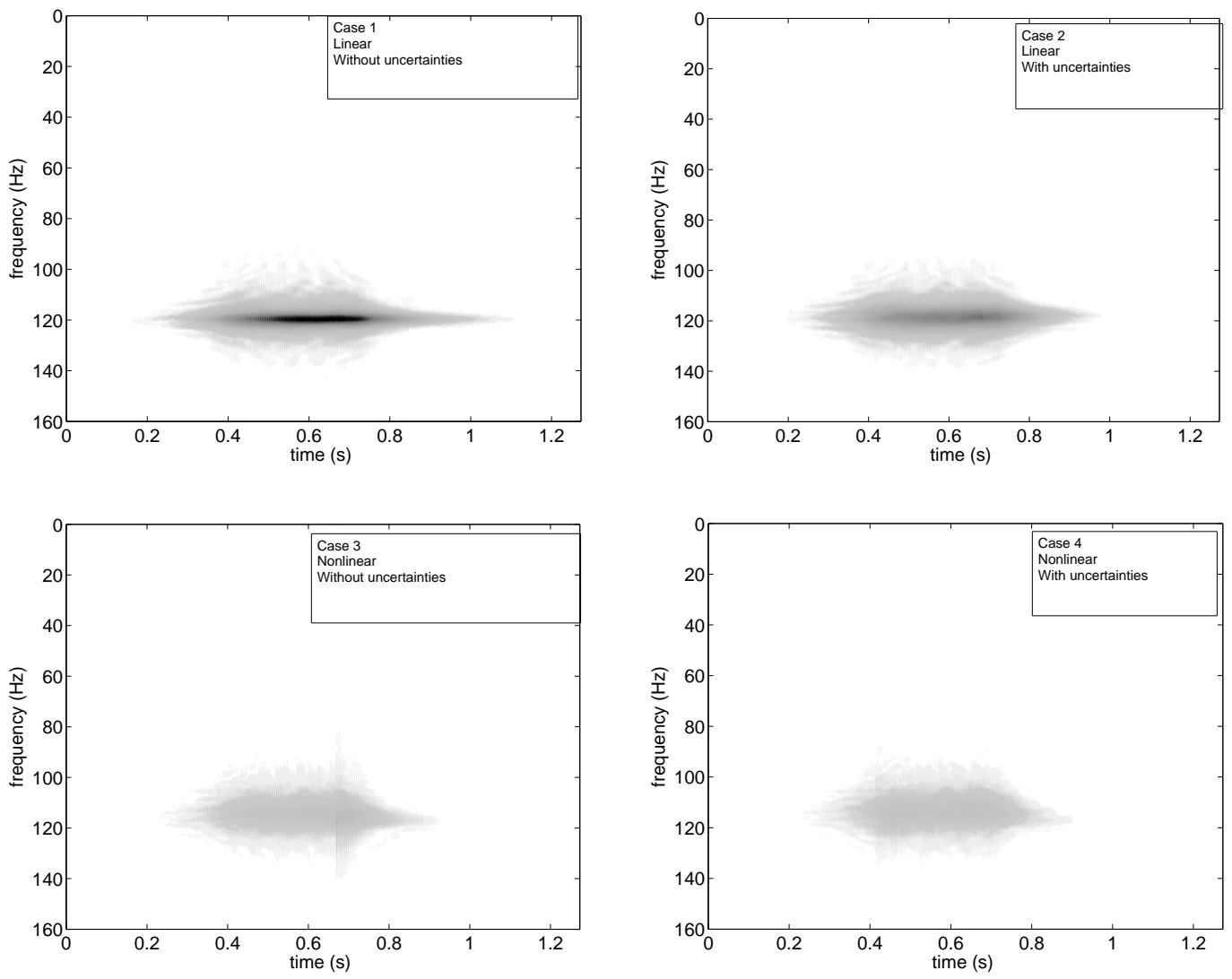

Figure 18. Graphs of functions $(\nu, t) \mapsto\left|s_{U_{o b s}}(\nu, t)\right|$ for cases 1 and 2 (upper graphs) and for cases 3 and 4 (lower graphs) 

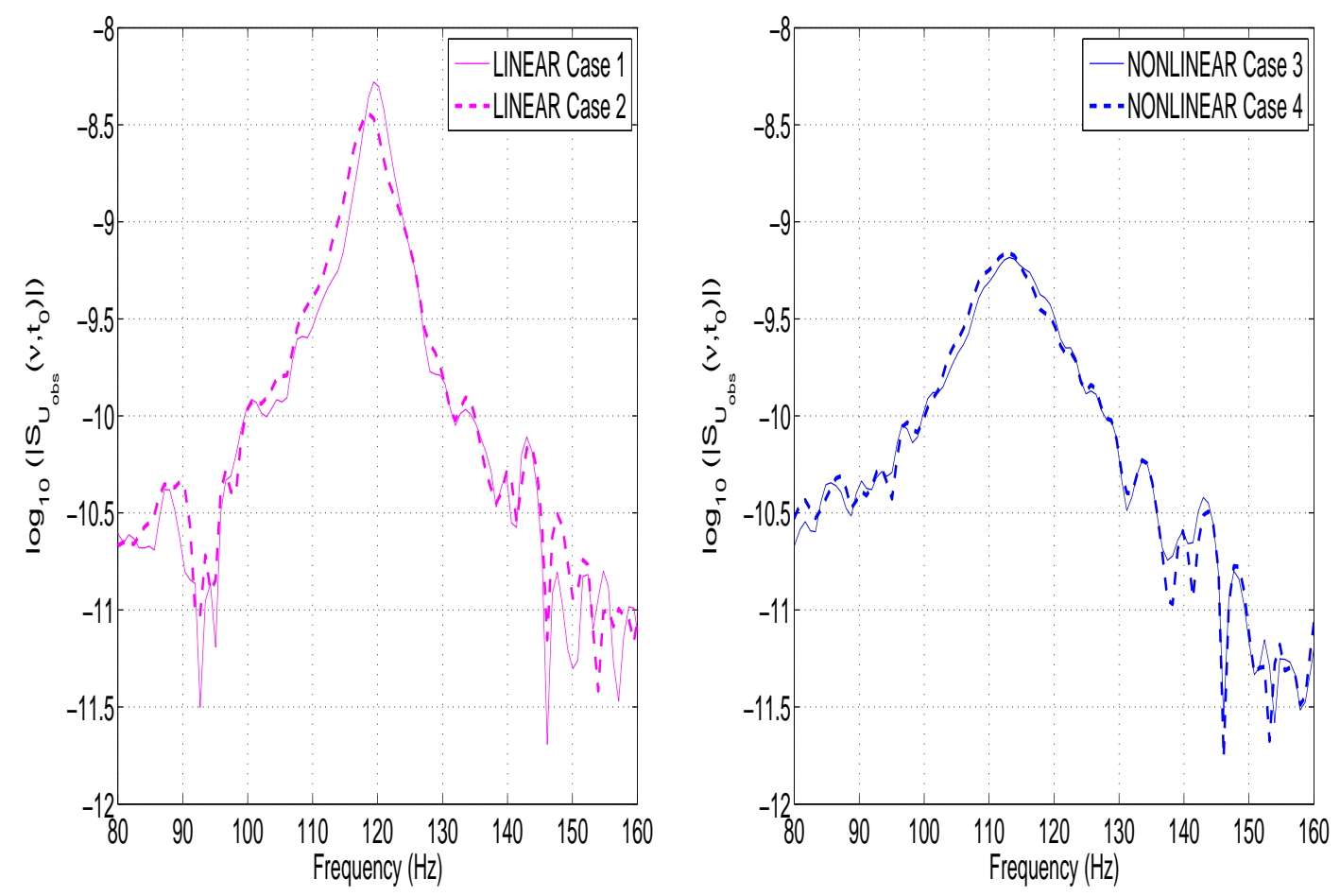

Figure 19. Graphs of $\nu \mapsto \log _{10}\left(\left|s_{U_{\text {obs }}}\left(\nu, t_{0}\right)\right|\right)$ without and with random uncertainties (thin line and thick dashed line): linear case (left graph) and nonlinear case (right graph).

From here on, for $t \in[0, T]$, for $\theta \in \Theta$ and for $\theta^{\prime} \in \Theta^{\prime}$, a realization of the stochastic process $U_{o b s}(t)$ is denoted by $U_{o b s}\left(t ; \theta, \theta^{\prime}\right)$. To better understand and analyze the effects of random uncertainties on the nonlinear stochastic response, a spectral analysis of the nonstationary observation $U_{o b s}(t)$ is next. To this end, let

$$
S_{U_{o b s}}(\nu, t ; \theta)=\frac{1}{2 \pi} \int_{0}^{T} e^{-2 i \pi \nu t^{\prime}} R_{U_{o b s}}\left(t^{\prime}, t ; \theta\right) d t^{\prime},
$$

be a sample of the complex-valued random instantaneous spectral density function for $\nu$ in $\mathbb{B}_{\nu}$, for $t$ in $[0, T]$ and for $\theta$ in $\Theta$. In this equation, for all fixed $t$ and $t^{\prime}, \theta \mapsto R_{U_{o b s}}\left(t, t^{\prime} ; \theta\right)$ is the real-valued random variable defined by

$$
R_{U_{o b s}}\left(t, t^{\prime} ; \theta\right)=\int_{\Theta^{\prime}} U_{o b s}\left(t ; \theta, \theta^{\prime}\right) U_{o b s}\left(t^{\prime} ; \theta, \theta^{\prime}\right) d \mathcal{P}^{\prime}\left(\theta^{\prime}\right)
$$


Therefore, $\left\{S_{U_{\text {obs }}}(\nu, t), \nu \in \mathbb{B}_{\nu}, t \in[0, T]\right\}$ is a second-order stochastic process defined on probability space $(\Theta, \mathcal{T}, \mathcal{P})$.
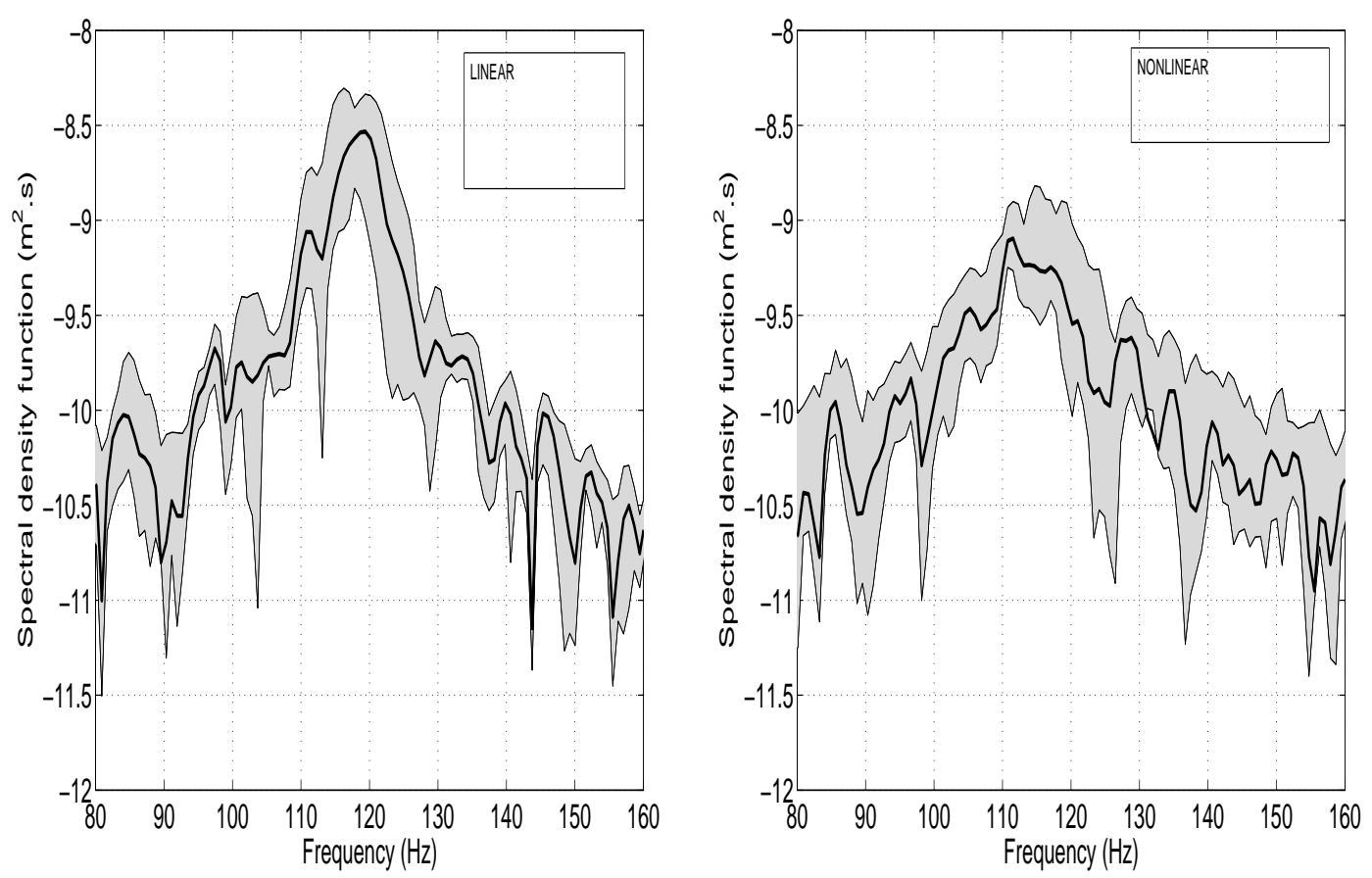

Figure 20. Confidence region: graph of $\nu \mapsto \log _{10}\left(\mathbb{E}\left\{\left|S_{U_{\text {obs }}}\left(\nu, t_{0}\right)\right|\right\}\right)$ (thick dashed line) and graph of the confidence region of $\nu \mapsto \log _{10}\left(\left|S_{U_{o b s}}\left(\nu, t_{0}\right)\right|\right)$ (grey region). Linear case (left graph) and nonlinear case (right graph).

Figure 20 shows the graph of the confidence region calculated with a probability level $P_{c}=0.95$ of the random stochastic process $\nu \mapsto \log _{10}\left(\left|S_{U_{\text {obs }}}\left(\nu, t_{0}\right)\right|\right)$ for the linear case (left graph) and for the nonlinear case (right graph). Note that the computations have been carried out for $77 \times 77=5929$ realizations $\left(\theta, \theta^{\prime}\right)$. It can be seen that the peak response corresponding to the nonlinear case occurs for a lower frequency and with a smaller magnitude than its linear counterpart. Furthermore, the confidence region related to the nonlinear case is broader, demonstrating that the response prediction in the nonlinear case is less robust with respect to uncertainties than in the linear case. 


\section{Conclusion}

The paper has presented an experimental validation of an advanced computational method for analyzing the nonlinear post-buckling behavior of a geometrically nonlinear thin shell structure in presence of uncertainties. The experimental static nonlinear response of a very thin cylindrical shell with respect to the intensity of a static shear load constitute the experimental data. Firstly, the mean nonlinear computational model has been used for generating the nonlinear static reference response from which the POD basis has been constructed. The nonlinear stochastic computational model has then been constructed and experimentally identified in the goal to capture the reference response for the best. For this kind of very thin structures investigated, the sensitivity of the mechanical behavior is very high. The updating is considered as accurate when the experimental response belongs to the confidence region. It can be concluded that the computational model which has experimentally been identified yields a slight overestimation of the static nonlinear response in the linear range, while the buckling and the postbuckling ranges are particularly sensitive to uncertainties and are accurately predicted by the identified stochastic computational model. By increasing the shear load, it has been shown that the computational model predicts a range for which a linear elastic stiffening is observed, for which the robustness to uncertainties lightly increases.

Then, the identified stochastic computational model has been used for predicting the nonlinear dynamical post-buckling response, under a stochastic excitation induced by a ground motion of the structure. The influence of geometrical nonlinearities has been analyzed through instantaneous spectral density functions. The presence of geometrical nonlinearities modifies the dynamical behavior (1) by inducing a local softening effect and a decrease of the resonance magnitude; (2) by decreasing the robustness of the response predictions with respect to uncertainties. 


\section{References}

[1] J. Hutchinson, Buckling and initial postbuckling behaviour of oval cylindrical shells under axial compression., ASME Journal of Applied Mechanics 35 (1) (1968) 66-76.

[2] R. Tennyson, K. Chan, Buckling of imperfect sandwich cylinders under axial compression., International Journal of Solids and Structures 26 (1990) 1017-1036.

[3] P. Mandal, R. Calladine, Buckling of thin cylindrical shells under axial compression., International Journal of Solids and Structures 37 (33) (2000) 4509-4525.

[4] H. Huang, Q. Han, Research on nonlinear postbuckling of functionally graded cylindrical shells under radial loads, Computers \& Structures 92 (6) (2010) 1352-1357.

[5] L. Chen, J. Rotter, Buckling of anchores cylindrical shells of uniform thickness under wind load., Engineering Structures 41 (2012) 199-208.

[6] S. Matsuura, H. Nakamura, K. Kokubo, S. Ogiso, H. Ohtsubo, Shearbending buckling analyses of fast breeder reactor main vessels, Nuclear Engineering and Design 153 (1995) 305-317.

[7] J. Okada, K. Iwata, K. Tsukimori, T. Nagata, An evaluation method for elastic-plastic buckling of cylindrical shells under shear forces., Nuclear Engineering and Design 157 (1995) 65-79.

[8] G. Michel, A. Combescure, J.-F. Jullien, Finite element simulation of dynamic buckling of cylinders subjected to periodic shear, Thin-Walled Structures 36 (2000) 111-135.

[9] G. Michel, A. Limam, J.-F. Jullien, Buckling of cylindrical shells under static and dynamic shear loading, Engineering Structures 22 (2000) 535543.

[10] W. Schneider, Y. Ribakov, Collapse analysis of thin walled cylindrical steel shell subjected to constant shear stress, Computers \& Structures 82 (29-30) (2004) 2463-2470. 
[11] Z. Qiu, Convex models and interval analysis method to predict the effect of uncertain-but-bounded parameters on the buckling of composite structures, Computer Methods in Applied Mechanics and Engineering 194 (18-20) (2004) 2175-2189.

[12] I. Elishakoff, Uncertain buckling: its past, present and future, International Journal of Solids and Structures 37 (46-47) (2000) 6869-6889.

[13] I. Elishakoff, B. Kriegesmann, R. Rolfes, Optimization and antioptimization of buckling load for composite cylindrical shells under uncertainties, AIAA Journal 50 (7) (2012) 1513-1524.

[14] H. Pradlwarter, G. Schueller, C. Schenk, A computational procedure to estimate the stochastic dynamic response of large non-linear FE-models, Computer Methods in Applied Mechanics and Engineering 192 (7-8) (2003) 777-801.

[15] H. J. Pradlwarter, G. I. Schueller, Reliability of deterministic non-linear systems subjected to stochastic dynamic excitation, International Journal for Numerical Methods in Engineering 85 (9) (2011) 1160-1176.

[16] C. Schenk, G. Schuëller, Buckling analysis of cylindrical shells with random geometric imperfections, International Journal of Nonlinear Mechanics 38 (7) (2003) 1119-1132.

[17] M. Broggi, G. Schuëller, Efficient modeling of imperfections for buckling analysis of composite cylindrical shells., Engineering Structures 33 (2011) 1796-1806.

[18] C. Schenk, G. Schuëller, Buckling analysis of cylindrical shells with cutouts including random boundary and geometric imperfections, Computer Methods in Applied Mechanics and Engineering 196 (35-36) (2007) 3424-3434.

[19] V. Papadopoulos, G. Stefanou, M. Papadraakis, Buckling analysis of imperfect shells with stochastic non-gaussian materials and thickness properties, International Journal of Solids and Structures 46 (14-15) (2009) 2800-2808. 
[20] D. Schillinger, V. Papadopoulos, M. Bischoff, M. Papadrakakis, Buckling analysis of imperfect i-sections beam-columns with stochastic shell finite elements, Computational Mechanics 46 (3) (2010) 495-510.

[21] C. Soize, Stochastic Models of Uncertainties in Computational Mechanics, Lecture Notes in Engineering Mechanics 2, American Society of Civil Engineers (ASCE), 2012.

[22] M.-P. Mignolet, C. Soize, Stochastic reduced order models for uncertain geometrically nonlinear dynamical systems, Computer Methods in Applied Mechanics and Engineering 197 (2008) 3951-3963.

[23] L. Sirovich, Turbulence and the dynamics of coherent structures , Quarterly of Applied Mathematics 45 (3) (1987) 561-571.

[24] R. Sampaio, C. Soize, Remarks on the efficiency of pod for model reduction in non-linear dynamics of continuous elastic systems, International Journal for Numerical Methods in Engineering 72 (1) (2007) 22-45.

[25] M. Azeez, A.-F. Vakakis, Proper orthogonal decomposition (POD) of a class of vibroimpact oscillations, Journal of Sound and Vibration 240 (5) (2001) 859-889.

[26] C. Desceliers, C. Soize, S. Cambier, Non-parametric - parametric model for random uncertainties in nonlinear structural dynamics: Application to earthquake engineering., Earthquake Engineering and Structural Dynamics 33 (2003) 315-327.

[27] A. Batou, C. Soize, Identification of stochastic loads applied to a nonlinear dynamical system using an uncertain computational model and experimental responses, Computational Mechanics 43 (4) (2009) 559571.

[28] K. Kim, A. Radu, M.-P. Mignolet, Nonlinear reduced order modeling of isotropic and functionally graded plates, International Journal of NonLinear Mechanics (in press).

[29] M.-P. Mignolet, A. Przekop, S. Rizzi, S. Spottswood, A review of indirect/non-intrusive reduced order modeling of nonlinear geometric structures., Journal of Sound and Vibration 332 (10) (2013) 2437-2460. 
[30] A. Muryavov, S. Rizzi, Determination of nonlinear stiffness with application to random vibration of geometrically nonlinear structures, Computers \& Structures 81 (2003) 1513-1523.

[31] E. Capiez-Lernout, C. Soize, M.-P. Mignolet, Computational stochastic statics of an uncertain curved structure with geometrical nonlinearity in three-dimensional elasticity., Computational Mechanics 49 (1) (2012) 87-97.

[32] R. Murthy, X. Wang, R. Perez, M.-P. Mignolet, L. Richter, Uncertaintybased experimental validation of nonlinear reduced order models, Journal of Sound and Vibration 331.

[33] R. Ohayon, C. Soize, Structural acoustics and vibration, Academic Press, San Diego, London, 1998.

[34] C. Soize, Random matrix theory for modeling random uncertainties in computational mechanics, Computer Methods in Applied Mechanics and Engineering 194 (12-16) (2005) 1333-1366.

[35] P. Kree, C. Soize, Mathematics of random phenomena random vibrations of mechanical structures, D. Reidel Pub. Co., 1986.

[36] G. Saragoni, G. Hart, Simulation of artificial earthquakes, Earthquake Engineering and Structural Dynamics 2 (3) (1974) 249-267.

[37] D. Boore, Simulation of ground motion using the stochastic method, Pure and Applied Geophysics 160 (3-4) (2003) 635-676.

[38] H. Kanai, Semi-empirical formula for the seismic characteristics of the ground motion, Bulletin of the Earthquake Research Institute 35 (1957) 309-325.

[39] K. Bathe, Finite Element Procedures in Engineering Analysis, PrenticeHall, 1982.

[40] M. Crisfield, Non-linear finite element analysis of solids and structures, Vol. 1 : essentials, John Wiley and Sons, Chichester, 1997.

[41] G. Michel, Flambage de coques minces cylindriques sous un chargement dynamique de cisaillement, Ph.D. thesis, INSA Lyon (1997). 
[42] M. Meyer, H. Matthies, Efficient model reduction in non-linear dynamics using the karhunen-loeve expansion and dual-weighted-residual methods, Computational Mechanics 31 (1-2) (2003) 179-191.

[43] M. Amabili, C. Touze, Reduced-order models for nonlinear vibrations of fluid-filled circular cylindrical shells: Comparison of pod and asymptotic nonlinear normal modes methods, Journal of Fluids and Structures 23 (6) (2007) 885-903.

[44] S. Chaturantabut, D. C. Sorensen, A state space error estimate for poddeim nonlinear model reduction, SIAM Journal on numerical analysis 50 (1) (2012) 46-63.

[45] J. J. Dongarra, J. Bunch, C. Moler, G. Stewart, LINPACK Users Guide, Philadelphia, PA: SIAM Publications, 1978.

[46] M. Arnst, R. Ghanem, S. Masri, Maximum entropy approach to the identification of stochastic reduced-order models of nonlinear dynamical systems, Aeronautical Journal 114 (1160) (2010) 637-650.

[47] M. Beer, M. Liebscher, Designing robust structures - A nonlinear simulation based approach, Computers \& Structures 86 (10) (2008) 1102-1122.

[48] H. A. Jensen, M. Beer, Discrete-continuous variable structural optimization of systems under stochastic loading, Structural Safety 32 (5) (2010) 293-304.

[49] C. Soize, E. Capiez-Lernout, J.-F. Durand, C. Fernandez, L. Gagliar dini, Probabilistic model identification of uncertainties in computational models for dynamical systems and experimental validation., Computer Mechanical Methods in Applied Mechanics and Engineering 98 (1) (2008) 150-163.

[50] R. Serfling, Approximation theorems of mathematical statistics, Wiley, New York, 1980.

[51] L. Ludeman, Fundamentals of Digital Signal Processing, Harper and Row, New York, 1986.

[52] C. Soize, Méthodes mathématiques en analyse du signal, Masson, 1993. 